\title{
STABILITY THEORY FOR PARAMETRIC GENERALIZED EQUATIONS AND VARIATIONAL INEQUALITIES VIA NONSMOOTH ANALYSIS
}

\author{
BORIS MORDUKHOVICH
}

\begin{abstract}
In this paper we develop a stability theory for broad classes of parametric generalized equations and variational inequalities in finite dimensions. These objects have a wide range of applications in optimization, nonlinear analysis, mathematical economics, etc. Our main concern is Lipschitzian stability of multivalued solution maps depending on parameters. We employ a new approach of nonsmooth analysis based on the generalized differentiation of multivalued and nonsmooth operators. This approach allows us to obtain effective sufficient conditions as well as necessary and sufficient conditions for a natural Lipschitzian behavior of solution maps. In particular, we prove new criteria for the existence of Lipschitzian multivalued and single-valued implicit functions.
\end{abstract}

\section{INTRODUCTION}

This paper is concerned with parametric generalized equations of the form

$$
0 \in f(p, z)+Q(z)
$$

where $f: \mathbb{R}^{k} \times \mathbb{R}^{n} \rightarrow \mathbb{R}^{m}$ is a given continuous function and $Q: \mathbb{R}^{n} \Rightarrow \mathbb{R}^{m}$ is a given multifunction of closed graph. Our primary goal is to study the dependence of the solution map

$$
\Sigma(p):=\left\{z \in \mathbb{R}^{n}: 0 \in f(p, z)+Q(z)\right\}
$$

on the parameter $p$ near a reference point. These questions are addressed to local sensitivity analysis of the generalized equation (1.1) under parameter perturbations.

The term "generalized equation" has been introduced by Robinson [28] who has considered some important classes of generalized equations (1.1) with multifunctions $Q$ of special structure. If $\Omega \subset \mathbb{R}^{n}$ is a convex set and

$$
Q(z)=N(z \mid \Omega):= \begin{cases}\left\{z^{*} \in \mathbb{R}^{n}:\left\langle z^{*}, z-z^{\prime}\right\rangle \leq 0 \forall z^{\prime} \in \Omega\right\} & \text { for } z \in \Omega, \\ \varnothing & \text { otherwise }\end{cases}
$$

Received by the editors June 29, 1992.

1991 Mathematics Subject Classification. Primary 49J40, 49J52, 90C31; Secondary 58C06, $58 \mathrm{C} 15,90 \mathrm{C} 33$.

Key words and phrases. Generalized equations, variational inequalities, stability and sensitivity, nonsmooth analysis, generalized differentiation.

This research was supported by the National Science Foundation under grant DMS-9006224. 
is the normal-cone operator in the sense of convex analysis [32], then the generalized equation (1.1) is reduced to the parametric variational inequality

$$
\text { find } z \in \Omega \text { such that }\left\langle f(p, z), z-z^{\prime}\right\rangle \geq 0 \text { for each } z^{\prime} \in \Omega
$$

which is of particular interest for applications. It is well known that models (1.1) and (1.3) cover a wide range of important problems in mathematical programming, complementarity, mathematical economics, game theory, equilibria, etc.; see Robinson [30] and Harker and Pang [8] for discussions and many examples.

As in most cases of mathematical models, sensitivity analysis is an important part in the solution of generalized equations and variational inequalities. One of the principal questions here is solution stability under parameter perturbations. For models (1.1) and (1.3), stability questions are important not only for a better understanding of the solution behavior with respect to perturbations, but also for constructing effective numerical algorithms to solve the problems; see, e.g., $[8,16,24,30]$.

There are many publications devoted to sensitivity analysis of variational inequalities in forms (1.1) or (1.3); few of them also consider more general cases of (1.1). We refer the reader to $[6-8,10,15,16,23,24,26,28-31,37]$ and bibliographies therein. Most works in the area conduct a local sensitivity analysis of a given solution point under Robinson's strong regularity condition [29] or some of its modifications; see $\S 7$ for the definition and discussions.

The strong regularity condition ensures the uniqueness of the solution to (1.1) around the reference point and its locally Lipschitzian dependence on the parameter. In general, the solution map (1.2) is not single-valued and turns out to be a multifunction on the parameter. So, in the absence of strong regularity, the question arises about Lipschitzian stability of the solution map (1.2) in an appropriate sense.

Some results in this direction have been already obtained in the first Robinson paper on generalized equations [28] where the upper Lipschitzian property of the solution map (1.2) has been studied. This property describes a kind of unilateral Lipschitzian behavior of multifunctions which is not reduced to the classical local Lipschitzness in the case of single-valued maps. The recent papers $[7,10,15,24,26]$ contain new conditions ensuring an upper Lipschitzian type of behavior for solution maps to variational inequalities.

Traditional approaches to the sensitivity and stability questions in generalized equations/variational inequalities are based on fixed point arguments, contractive mapping principles, and related tools in analysis and topology; see, e.g., [6, $8,10,28-31]$. A number of new results for parametric variational inequalities have been recently obtained in [7, 24] by using degree theory arguments.

When $Q(z) \equiv 0$, the generalized equation (1.1) is converted to a system of standard (ordinary) parametric equations. In this case, (1.2) defines an implicit function (or multifunction) depending on the parameter $p$. In general, the form of (1.1) successing the form of ordinary equations is helpful for extending some methods and results to the generalized case (1.1) by analogy with the classical setting. Note also that there is a way to obtain an ordinary parametric equation equivalent to the variational inequality (1.3) by using the normal (Minty) map; see, e.g., [10 and 31]. This map is related to the projection operator on the set $\Omega$ and turns out to be nondifferentiable even in the case of smooth functions $f$. 
In this paper, we consider generalized equations and variational inequalities in Robinson's form (1.1) which is proved to be convenient for the theory and applications. In many cases where formalism (1.1) seems particularly useful, the vector function $f$ is sufficiently smooth while the multivalued part $Q$ accumulates the nonsmoothness ("corners") inherent in the problem. Such nonsmoothness is a natural feature of generalized equations and cannot be avoided under any transformations. So, the generalized equation model (1.1) appears to be a real "nonsmooth" problem which might be studied by methods of nonsmooth analysis.

The heart of nonsmooth analysis is generalized differentiation of nondifferentiable (in classical senses) functions and set-valued mappings. There are various concepts of generalized derivatives which are useful in the optimization theory, related areas, and applications; we refer to the monographs $[2,4,19$, 36] and citations therein. Some of these concepts have been recently used in the sensitivity analysis of nonsmooth equations in standard or generalized forms. Namely, Robinson [31] employs the concept of strong Bouligand (B-) derivative of nonsmooth functions for obtaining Lipschitzian implicit-function theorems in ordinary equations and variational inequalities. King and Rockafellar [10] and Pang [24] provide sufficient conditions for upper Lipschitzian properties of solution maps using related constructions for multifunctions connected with the contingent derivative.

This paper is devoted to a systematic study of the Lipschitzian stability for parametric generalized equations in form (1.1) where $Q$ is permitted to be an arbitrary closed-graph multifunction. In general, the solution map (1.2) to (1.1) is multivalued. In our stability analysis, we concentrate on Lipschitzian properties of (1.2) which generalize the classical local Lipschitzness around the reference point (i.e., in some of its neighborhood).

The main attention will be paid to the so-called pseudo-Lipschitzian property of the solution map (1.2) around a given point in its graph. This property of multifunctions introduced by Aubin [1] appears to be equivalent to the well-known openness and metric regularity properties of the inverse operators. For the case of single-valued maps, the pseudo-Lipschitzian property coincides with the usual Lipschitz continuity. Moreover, if a multifunction is pseudoLipschitzian and monotone, then it must be locally single-valued. We refer to $\S 3$ for more details about pseudo-Lipschitzian and related properties of multifunctions.

In [1], Aubin studies the pseudo-Lipschitzian behavior of solution maps to perturbed convex minimization problems. Rockafellar's thorough study [34] is addressed to the Lipschitzian stability of general constraint systems including solution maps to parametric generalized equations. Both papers $[1,34]$ and some other publications provide sufficient conditions for the pseudo-Lipschitzian property of solution maps employing Clarke's derivative-like constructions in nonsmooth analysis; see [4].

For parametric systems like (1.2), the conditions obtained are expressed in terms of Clarke's normal (or tangent) cone to the graph of $Q$ and turn out to be too restrictive for applications in many important problems. They actually require certain smoothness of the multifunction $Q$ and do not cover at all the cases of variational inequalities and complementarity problems. The reader can find more details and references in $\S 3$ and Remark 4.9. 
In this paper we employ other derivative-like constructions in nonsmooth analysis which were first used in Mordukhovich [17, 18] for obtaining necessary optimality conditions in nonsmooth problems. These objects are usually much smaller than Clarke's ones and take nonconvex values. They always possess robust (stability) properties with respect to perturbations and enjoy a rich calculus important for applications. We refer the reader to the next section for more information.

The main construction used in this paper for studying the Lipschitzian stability is the coderivative of multifunctions. This object appears to be an adequate tool for complete characterizations of locally Lipschitz behavior for arbitrary closed-graph multifunctions in finite dimensions. One can find various criteria for pseudo-Lipschitzian and related properties of general multifunctions in Mordukhovich [20, 22, 23]. Some of these criteria are employed here for obtaining Lipschitzian stability results in parametric generalized equations and variational inequalities.

We have been concerned with this topic in [23] by reducing (1.1) to more general nonsmooth constraint systems. Here we develop another approach to Lipschitzian stability of (1.1) and (1.3) which brings together criteria in nonsmooth analysis and linearization/strong approximation procedures in the spirit of Robinson [28, 29, 31]. In this way, we obtain new necessary and sufficient conditions as well as refined sufficient conditions for Lipschitzian stability of the solution maps to (1.1) and (1.3) which essentially strengthen some of the results in [23] and other publications.

The organization of the paper is as follows. Section 2 contains some background material on generalized differentiation of multifunctions and nonsmooth mappings including first and second order subdifferentials for extendedreal-valued functions. We provide main calculus rules which are broadly used in what follows.

In $\S 3$ we consider Lipschitzian properties of general multifunctions and also a special class of multivalued operators whose graphs are Lipschitzian manifolds in the sense of Rockafellar [35]. This class includes, in particular, all monotone operators and covers a wide range of applications. We present useful characterizations of the locally Lipschitz behavior of multifunctions in terms of the nonconvex coderivative introduced as well as their "strictly smooth" analogues in terms of the Clarke counterpart.

Section 4 is devoted to the stability theory for linear systems (1.1) and (1.3). Using the derivative-like constructions in $\S 2$, we obtain necessary and sufficient conditions for the pseudo-Lipschitzian property of the solution map (1.2) in the general case of (1.1) and for special representations of the multifunction $Q$.

In $\S 5$ we deal with nonlinear generalized equations (1.1) where the function $f$ is smooth in the decision variables. Using a recent result in Dontchev and Hager [6], we reduce the study of the pseudo-Lipschitzian property for the solution map (1.2) to the corresponding property of the solution map to a linearized system. Then we obtain effective conditions for the Lipschitzian stability of (1.1) and (1.3) in the smooth case employing criteria in $\S 4$.

In $\S 6$ we develop the latter linearization (approximation) procedure to the case of nonsmooth generalized equations with functions $f$ admitting a strong approximation in Robinson's sense [31]. We obtain sufficient conditions for the pseudo-Lipschitzian property of the solution map (1.2) in terms of our 
generalized derivatives for the initial data in (1.1) and (1.3). For the case of $Q(z) \equiv 0$ in (1.1), the results obtained provide new conditions ensuring the pseudo-Lipschitzness of implicit multifunctions.

The concluding $\S 7$ deals with generalized equations (1.1) where $Q$ is a monotone operator. This includes the case of variational inequalities (1.3) due to the well-known monotonicity of the normal-cone multifunction $Q(z)=N(z \mid \Omega)$ for any convex set $\Omega$. For such a class of monotone equations, we obtain refined sufficient conditions as well as necessary and sufficient conditions ensuring the local single-valuedness and Lipschitz continuity of solution maps. In particular, the results obtained imply necessary and sufficient conditions for Robinson's strong regularity in a rather general setting.

In this paper we basically use standard notation; see, e.g., [4, 22, 32]. For a multifunction $\Phi: \mathbb{R}^{n} \Rightarrow \mathbb{R}^{m}$, we denote by

$$
\begin{gathered}
\operatorname{Dom} \Phi=\{x: \Phi(x) \neq \varnothing\}, \quad \operatorname{Im} \Phi=\{y \in \Phi(x): x \in \operatorname{Dom} \Phi\}, \\
\operatorname{Ker} \Phi=\{x: 0 \in \Phi(x)\}
\end{gathered}
$$

its domain, image, and kernel. The set

$$
\begin{aligned}
\limsup _{x \rightarrow \bar{x}} \Phi(x):=\left\{y \in \mathbb{R}^{m}:\right. & \exists \text { sequences } x_{k} \rightarrow \bar{x}, y_{k} \rightarrow y \\
& \text { with } \left.y_{k} \in \Phi\left(x_{k}\right) \forall k=1,2, \ldots\right\}
\end{aligned}
$$

is called the Kuratowski-Painlevé upper limit of the multifunction $\Phi(x)$ as $x \rightarrow$ $\bar{x}$.

If $\varphi: \mathbb{R}^{n} \rightarrow \overline{\mathbb{R}}=[-\infty, \infty]$ is an extended-real-valued function, then its lower and upper limits are denoted, respectively, by $\underline{\lim }$ and $\varlimsup$. The symbol $x(\in \Omega) \rightarrow \bar{x}$ means that $x \rightarrow \bar{x}$ with $x \in \Omega$. The adjoint (transpose) matrix to $A$ is denoted by $A^{*}$.

\section{Generalized DifFERENTIATION OF MULTIVALUED AND NONSMOOTH OPERATORS}

This section is devoted to reviewing some results in nonsmooth analysis which are broadly used in the main body of the paper. We develop an approach to generalized differentiation of nonsmooth mappings and multifunctions based on the concepts in Mordukhovich [17-19]. Let us begin with the definition of a normal cone to an arbitrary set in finite dimensions.

Let $\Omega$ be a nonempty closed set in $\mathbb{R}^{n}$ and let

$$
P(x, \Omega):=\{\omega \in \Omega:\|x-\omega\|=\operatorname{dist}(x, \Omega)\}
$$

denotes the set of best approximations of $x$ in $\Omega$ with respect to the Euclidean distance $\operatorname{dist}(x, \Omega)$. The closed cone

$$
N(\bar{x} \mid \Omega):=\limsup _{x \rightarrow \bar{x}}[\operatorname{cone}(x-P(x, \Omega))]
$$

is called the normal cone to $\Omega$ at the point $\bar{x} \in \Omega$.

If $\Omega$ is a convex set, then the normal cone introduced coincides with the normal cone of convex analysis. In general, the normal cone (2.1) may be nonconvex in very simple situations. For example, if $\Omega=\operatorname{gph}|x|$ with $x \in \mathbb{R}$, then one easily has

$$
N(0, \Omega)=\left\{(v, u) \in \mathbb{R}^{2}: u \leq-v \vee u=|v|\right\} .
$$


It turns out that the Clarke normal cone to $\Omega$ at $\bar{x}$ always coincides with the convex closure of (2.1), i.e.,

$$
N_{C}(\bar{x} \mid \Omega)=\operatorname{clco} N(\bar{x} \mid \Omega) .
$$

(This is actually the "proximal normal formula" in [4, Proposition 2.5.7].)

Let us observe that the taking of the convex closure as in (2.2) may dramatically enlarge the normal cone (2.1). In particular,

$$
N_{C}(0|\operatorname{gph}| x \mid)=\mathbb{R}^{2}
$$

for the set $\Omega$ in the example above. Such a picture is typical for sets which are locally represented as graphs of Lipschitz continuous functions; see the next section. Moreover, the convexity operation in (2.2) may spoil some nice properties enjoyed by the normal cone $(2.1)$. We refer to $[9,19,36]$ for more details.

For all $x \in \Omega$ let us consider the so-called Fréchet normal cone

$$
\widehat{N}(x \mid \Omega):=\left\{x^{*} \in \mathbb{R}^{n}: \varlimsup_{x^{\prime}(\in \Omega) \rightarrow x}\left\|x^{\prime}-x\right\|^{-1}\left\langle x^{*}, x^{\prime}-x\right\rangle \leq 0\right\}
$$

which is always convex and coincides (in finite dimensions) with the polar to (Bouligand) contingent cone; see, e.g., [2, Chapter 4]. The following important representation was first obtained in Kruger and Mordukhovich [11, 12]; cf. also [9, Theorem 1] and [19, Theorem 1.1].

Proposition 2.1. One has

$$
N(\bar{x} \mid \Omega)=\limsup _{x(\in \Omega) \rightarrow \bar{x}} \widehat{N}(x \mid \Omega) .
$$

for any closed sets $\Omega \subset \mathbb{R}^{n}$ and points $\bar{x} \in \Omega$.

It follows from (2.2) and Proposition 2.1 that

$$
\widehat{N}(\bar{x} \mid \Omega) \subset N(\bar{x} \mid \Omega) \subset N_{C}(\bar{x} \mid \Omega) .
$$

The set $\Omega$ is called regular at the point $\bar{x} \in \Omega$ if the three normal cones in (2.4) coincide. It happens for all convex sets as well as for "smooth" sets which are locally described by equality and inequality type constraints with smooth functions under the well-known Mangasarian-Fromovitz constraint qualification; see [19, Proposition 1.5 and 33, Theorem 2F]. On the other hand, this regularity is always broken for sets locally represented as graphs of nonsmooth Lipschitzian functions as in the example above. But such sets naturally appear in the following coderivative constructions which are realizations of the geometric (graphical) approach to differentiation going back to Fermat.

Let $\Phi: \mathbb{R}^{n} \Rightarrow \mathbb{R}^{m}$ be a multifunction of closed graph. The multifunction $D^{*} \Phi(\bar{x}, \bar{y}): \mathbb{R}^{m} \Rightarrow \mathbb{R}^{n}$ defined by

$$
D^{*} \Phi(\bar{x}, \bar{y})\left(y^{*}\right):=\left\{x^{*} \in \mathbb{R}^{n}:\left(x^{*},-y^{*}\right) \in N((\bar{x}, \bar{y}) \mid \operatorname{gph} \Phi)\right\}
$$

is called the coderivative of $\Phi$ at the point $(\bar{x}, \bar{y}) \in \operatorname{gph} \Phi$. The symbol $D^{*} \Phi(\bar{x})\left(y^{*}\right)$ is used in $(2.5)$ when $\Phi$ is single-valued at $\bar{x}$.

If the normal cone $(2.1)$ in formula $(2.5)$ is replaced by the normal cones (2.2) or (2.3), then the constructions

$$
D_{C}^{*} \Phi(\bar{x}, \bar{y})\left(y^{*}\right):=\left\{x^{*} \in \mathbb{R}^{n}:\left(x^{*},-y^{*}\right) \in N_{C}((\bar{x}, \bar{y}) \mid \operatorname{gph} \Phi)\right\},
$$




$$
\widehat{D}^{*} \Phi(\bar{x}, \bar{y})\left(y^{*}\right):=\left\{x^{*} \in \mathbb{R}^{n}:\left(x^{*},-y^{*}\right) \in \widehat{N}((\bar{x}, \bar{y}) \mid \operatorname{gph} \Phi)\right\}
$$

are called, respectively, the Clarke coderivative and the contingent coderivative of $\Phi$ at $(\bar{x}, \bar{y})$.

The primary tool of the nonsmooth analysis employed in the paper is the coderivative (2.5). Let us consider some properties of this object important for our applications.

Proposition 2.2. For any multifunctions $\Phi$ and points $(\bar{x}, \bar{y}) \in \operatorname{gph} \Phi, \bar{y}^{*} \in$ Dom $D^{*} \Phi(\bar{x}, \bar{y})$, one has

$$
\begin{aligned}
& D^{*} \Phi(\bar{x}, \bar{y})\left(\bar{y}^{*}\right)=\limsup _{\substack{(x, y)(\in \operatorname{gph} \Phi) \rightarrow(\bar{x}, \bar{y}) \\
y^{*} \rightarrow \bar{y}^{*}}} D^{*} \Phi(x, y)\left(y^{*}\right), \\
& D^{*} \Phi(\bar{x}, \bar{y})\left(\bar{y}^{*}\right)=\limsup _{(x, y)(\in \operatorname{ggh} \Phi) \rightarrow(\bar{x}, \bar{y})} \widehat{D}^{*} \Phi(x, y)\left(y^{*}\right) .
\end{aligned}
$$

Proof. Property (2.8) follows directly from definitions (2.5) and (2.1). Representation (2.9) is implied by Proposition 2.1 with $\Omega=\operatorname{gph} \Phi$.

Remark 2.3. Let us observe that (2.8) means the robustness (stability) of the coderivative (2.5) with respect to perturbations of the initial data. This property is of great importance for various applications, especially to sensitivity analysis of perturbed problems. Note that such a robustness may be broken for the Clarke coderivative (2.6); see an example in Rockafellar [33, p. 22]. This robustness property is always broken for the contingent coderivative (2.7) of any multifunction $\Phi$ whose graph is a nonsmooth Lipschitzian manifold around $(\bar{x}, \bar{y})$; see Proposition 3.3. Representation (2.9) actually means that the coderivative (2.5) is a robust regularization of the contingent coderivative.

Remark 2.4. Note that the contingent coderivative (2.7) and the Clarke coderivative (2.6) are convex-valued and turn out to be dual constructions to, respectively, the contingent derivative and the Clarke (circatangent) derivative of $\Phi$ at $(\bar{x}, \bar{y})$ introduced by Aubin; see [2, Chapter 5]. In particular, the contingent coderivative can be represented in the form

$$
\widehat{D}^{*} \Phi(x, y)\left(y^{*}\right)=\left\{x^{*}:\left\langle x^{*}, v\right\rangle \leq\left\langle y^{*}, u\right\rangle \forall(v, u) \in \operatorname{gph} \widehat{D} \Phi(x, y)\right\}
$$

where the contingent derivative $\widehat{D} \Phi(x, y)$ is defined as

$$
\widehat{D} \Phi(x, y)(v):=\limsup _{h \rightarrow v, \tau \downarrow 0}\left[\frac{\Phi(x+\tau h)-y}{\tau}\right]
$$

in terms of the difference quotients; we refer to [1 and 36] for more details.

Being nonconvex-valued, the coderivative (2.5) is not dual to any tangentially generated derivative construction. However, according to (2.9)-(2.11) it admits an analytical representation in terms of limits of the difference quotients.

From (2.4) and (2.5)-(2.7) it follows that

$$
\widehat{D} \Phi(\bar{x}, \bar{y})\left(y^{*}\right) \subset D^{*} \Phi(\bar{x}, \bar{y})\left(y^{*}\right) \subset D_{C}^{*} \Phi(\bar{x}, \bar{y})\left(y^{*}\right) \quad \forall y^{*} \in \mathbb{R}^{m} .
$$

If all three coderivatives in (2.12) coincide, then the multifunction $\Phi$ is called differentially regular at $(\bar{x}, \bar{y})$. This is obviously equivalent to the regularity of the graph of $\Phi$ at $(\bar{x}, \bar{y})$. The next sufficient conditions for the differential regularity are derived from [19, Proposition 3.2 and Corollary 3.3.2]. 
Proposition 2.5. $\Phi$ is differentially regular at $(\bar{x}, \bar{y}) \in \operatorname{gph} \Phi$ if one of the following conditions holds:

(i) $\Phi: \mathbb{R}^{n} \Rightarrow \mathbb{R}^{m}$ is a multifunction of convex graph. In this case one has

$$
\begin{aligned}
D^{*} \Phi(\bar{x}, \bar{y})\left(y^{*}\right) & =\left\{x^{*} \in \mathbb{R}^{n}:\left\langle x^{*}, \bar{x}\right\rangle-\left\langle y^{*}, \bar{y}\right\rangle\right. \\
& \left.=\max \left[\left\langle x^{*}, x\right\rangle-\left\langle y^{*}, y\right\rangle \mid(x, y) \in \operatorname{gph} \Phi\right]\right\} .
\end{aligned}
$$

(ii) $\Phi=f: \mathbb{R}^{n} \rightarrow \mathbb{R}^{m}$ is single-valued around $\bar{x}$ and strictly differentiable at $\bar{x}$ with the Jacobian $\nabla f(\bar{x}) \in \mathbb{R}^{m \times n}$, i.e.,

$$
\lim _{x, x^{\prime} \rightarrow \bar{x}}\left[\frac{f(x)-f\left(x^{\prime}\right)-\nabla f(\bar{x})\left(x-x^{\prime}\right)}{\left\|x-x^{\prime}\right\|}\right]=0 .
$$

In this case one has

$$
D^{*} f(\bar{x})\left(y^{*}\right)=\left\{(\nabla f(\bar{x}))^{*} y^{*}\right\} \quad \forall y^{*} \in \mathbb{R}^{m} .
$$

It turns out that the property of differential regularity always fails for a broad class of multifunctions whose graphs are nonsmooth Lipschitzian manifolds; see the next section (Propositions 3.1 and 3.3) for more details. Moreover, the coderivatives (2.5) and (2.6) are different in dimensions for such multifunctions; in particular, the inclusion $\operatorname{co} D^{*} \Phi(\bar{x}, \bar{y})\left(y^{*}\right) \subset D_{C}^{*} \Phi(\bar{x}, \bar{y})\left(y^{*}\right)$ is proper.

Now let us consider an extended-real-valued function $\varphi: \mathbb{R}^{n} \rightarrow \overline{\mathbb{R}}$ and define its first and second order subdifferentials associated with the coderivative (2.5). Let

$$
\Phi(x)=E_{\varphi}(x):=\{\mu \in \mathbb{R}: \mu \geq \varphi(x)\}
$$

be a multifunction with $\operatorname{gph} \Phi=\operatorname{epi} \varphi$. The set

$$
\begin{aligned}
\partial^{-} \varphi(\bar{x}): & =D^{*} E_{\varphi}(\bar{x}, \varphi(\bar{x}))(1) \\
& =\left\{x^{*} \in \mathbb{R}^{n}:\left(x^{*},-1\right) \in N((\bar{x}, \varphi(\bar{x})) \mid \operatorname{epi} \varphi)\right\}
\end{aligned}
$$

is called the (first order) subdifferential of $\varphi$ at $\bar{x} \in \operatorname{dom} \varphi:=\left\{x \in \mathbb{R}^{n}:|\varphi(x)|<\right.$ $\infty\}$. If $x \notin \operatorname{dom} \varphi$, then we put $\partial^{-} \varphi(x):=\varnothing$.

Let $\bar{x} \in \operatorname{dom} \varphi$ and $\bar{y} \in \partial^{-} \varphi(\bar{x})$. The set-valued mapping $\partial^{2,-} \varphi(\bar{x}, \bar{y})$ : $\mathbb{R}^{n} \Rightarrow \mathbb{R}^{n}$ defined by

$$
\begin{aligned}
\partial^{2,-} \varphi(\bar{x}, \bar{y})(u) & :=\left(D^{*} \partial^{-} \varphi\right)(\bar{x}, \bar{y})(u) \\
& =\left\{v \in \mathbb{R}^{n}:(v,-u) \in N\left((\bar{x}, \bar{y}) \mid \operatorname{gph} \partial^{-} \varphi\right)\right\}
\end{aligned}
$$

is called the second order subdifferential of $\varphi$ at $\bar{x}$ relative to $\bar{y}$.

Similarly one can define the following constructions of the superdifferential and the second order superdifferential for $\varphi$ :

$$
\partial^{+} \varphi(\bar{x}):=-\partial^{-}(-\varphi)(\bar{x}), \quad \partial^{2,+} \varphi(\bar{x}, \bar{y})(u):=-\left(D^{*} \partial^{+} \varphi\right)(\bar{x}, \bar{y})(-u)
$$

which are also important in that unilateral analysis of nonsmooth functions $\varphi$ being essentially different from the subdifferential constructions.

Note that the subdifferential $\partial^{-} \varphi$ is reduced to the classical subdifferential of convex analysis if $\varphi$ is convex; $\partial^{+} \varphi$ is reduced to the classical superdifferential for concave functions. Both first order semidifferentials in (2.13) and (2.15) are set-valued generalizations of the strict differentiability: they are reduced to the single gradient vector $\nabla \varphi(\bar{x})$ for smooth (i.e., strictly differentiable at $\bar{x}$ ) functions $\varphi$. At the same time,

$$
\partial^{2,-} \varphi(\bar{x})(u)=\partial^{2,+} \varphi(\bar{x})(u)=\left\{\left(\nabla^{2} \varphi(\bar{x})\right)^{*} u\right\} \quad \forall u \in \mathbb{R}^{n}
$$

if $\varphi \in \mathbb{C}^{2}$ with the Hessian matrix $\nabla^{2} \varphi(\bar{x})$. 
Properties of the first order subdifferential (2.13) and related constructions have been studied in detail in Mordukhovich [17-20]. Various properties and applications of this subdifferential can be found in many publications where (2.13) is often used in some equivalent forms under different names (in particular, the approximate subdifferential and the set of basic or limiting subgradients). For more information we refer to [19, 20], Ioffe [9], and Rockafellar and Wets [36].

If $\varphi$ is lower semicontinuous (1.s.c.) around $\bar{x}$, then the subdifferential (2.13) is represented by [19, Theorem 2.2]

$$
\partial^{-} \varphi(\bar{x})=\limsup _{x \rightarrow \bar{x}, \varphi(x) \rightarrow \varphi(\bar{x})} \widehat{\partial}^{-} \varphi(x)
$$

where the construction

$$
\widehat{\partial}^{-} \varphi(x):=\left\{x^{*} \in \mathbb{R}^{n}: \underset{x^{\prime} \rightarrow x}{\lim }\left[\frac{\varphi\left(x^{\prime}\right)-\varphi(x)-\left\langle x^{*}, x^{\prime}-x\right\rangle}{\left\|x^{\prime}-x\right\|}\right] \geq 0\right\}
$$

is well known in nonsmooth analysis as the Fréchet subdifferential; it coincides with the subdifferential in the sense of viscosity solutions [5].

The function $\varphi$ is called subdifferentially regular if

$$
\partial^{-} \varphi(\bar{x})=\widehat{\partial}^{-} \varphi(\bar{x})
$$

(see $[19, \S 2.3])$. When $\varphi$ is Lipschitz continuous around $\bar{x}$, the regularity introduced coincides with the Clarke (subdifferential) regularity; cf. [4, §2.3] and $[33, \S 3]$. The class of subdifferentially regular functions includes all convex functions, smooth functions, "max functions", etc. On the other hand, there are simple (and important) nonsmooth functions which are not subdifferentially regular. For instance, $\varphi(x)=-|x|, x \in \mathbb{R}$, where $\partial^{-} \varphi(0)=\{-1,1\}$ but $\widehat{\partial}^{-} \varphi(0)=\varnothing$.

It is well known (see, e.g., [19, Theorem 2.1]) that

$$
\partial_{C} \varphi(\bar{x})=\operatorname{co} \partial^{-} \varphi(\bar{x})=\operatorname{co} \partial^{+} \varphi(\bar{x})
$$

for Clarke's generalized gradient [4] of any locally Lipschitzian function $\varphi$. At the same time, if one changes the coderivative (2.5) by the Clarke coderivative (2.6) in the second order subdifferential (2.14), then the construction obtained may be much bigger than the convex hull of (2.14). It happens because graphs of the first order subdifferential mappings for important classes of functions (e.g., for convex and saddle ones) are Lipschitzian manifolds. But Clarke's normal cone is always a linear subspace for such manifolds; we refer again to the next section. In particular, if $\varphi$ is a closed proper convex function, then the Clarke second order subdifferential

$$
\begin{aligned}
\partial_{C}^{2,-} \varphi(\bar{x}, \bar{y})(u):= & \left(D_{C}^{*} \partial \varphi\right)(\bar{x}, \bar{y})(u) \\
& =\left\{x^{*} \in \mathbb{R}^{n}:\left(x^{*},-u\right) \in N((\bar{x}, \bar{y}) \mid \operatorname{gph} \varphi)\right\}
\end{aligned}
$$

(cf. Aubin [1]) is an affine subspace in $\mathbb{R}^{n}$ and cannot actually reflect anything other than classical-like "two-sides" aspects of differentiation; see Corollary 3.14 .

Now let us consider some calculus rules for the coderivative (2.5) and the subdifferential (2.13) used in this paper. Note that results for the subdifferential 
(2.13) immediately imply the corresponding results for the normal cone (2.1) because

$$
N(\bar{x} \mid \Omega)=\partial^{-} \delta(\bar{x}, \Omega) \quad \text { at } \bar{x} \in \Omega
$$

where $\delta(\cdot, \Omega)$ is the indicator of $\Omega$, i.e., $\delta(x, \Omega):=0$ if $x \in \Omega$ and $\delta(x, \Omega)$ $:=\infty$ if $x \notin \Omega$. One can observe that the subdifferential regularity of the function $\delta(\cdot, \Omega)$ at $\bar{x}$ is equivalent to the regularity of the set $\Omega$ at this point.

The next result is crucial for the generalized differential calculus of nonconvexvalued constructions (2.1), (25), (2.13)-(2.15);

Proposition 2.6. Let $\Phi_{1}$ and $\Phi_{2}$ be closed-graph multifunctions from $\mathbb{R}^{n}$ into $\mathbb{R}^{m}$, and let $\bar{y} \in \Phi_{1}(\bar{x})+\Phi_{2}(\bar{x})$. Let us assume that the sets

$$
M(x, y):=\left\{\left(y_{1}, y_{2}\right) \in \mathbb{R}^{2 m}: y_{1} \in \Phi(x), y_{2} \in \Phi_{2}(x), y_{1}+y_{2}=y\right\}
$$

are bounded uniformly around $(\bar{x}, \bar{y})$ and one has

$$
D^{*} \Phi_{1}\left(\bar{x}, y_{1}\right)(0) \cap\left(-D^{*} \Phi_{2}\left(\bar{x}, y_{2}\right)(0)\right)=\{0\} \quad \forall\left(y_{1}, y_{2}\right) \in M(\bar{x}, \bar{y}) .
$$

Then

$$
\begin{aligned}
D^{*}\left(\Phi_{1}+\Phi_{2}\right)(\bar{x}, \bar{y})\left(y^{*}\right) \subset \bigcup\left[D^{*} \Phi_{1}\left(\bar{x}, y_{1}\right)\left(y^{*}\right)+\left(y_{1}, y_{2}\right)\right. \\
\left.\quad \in M(\bar{x}, \bar{y}) D^{*} \Phi_{2}\left(\bar{x}, y_{2}\right)\left(y^{*}\right)\right] .
\end{aligned}
$$

The inclusion in (2.21) under the assumptions made is proved in [21, Theorem 3.6] by using the metric approximation method which develops the technique in [17-19].

One can obtain various corollaries of Proposition 2.6 (including calculus rules for the first and second order semidifferentials (2.13)-(2.15)) considering special kinds of single-valued and multivalued mappings $\Phi_{i}$ and ensuring conditions (2.19) and (2.20). One such effective assertion is Corollary 3.6. The following result guarantees equality in (2.21) without regularity assumptions.

Corollary 2.7. Let $f: \mathbb{R}^{n} \rightarrow \mathbb{R}^{m}$ be a single-valued function strictly differentiable around $\bar{x}$ and let $\Phi: \mathbb{R}^{n} \Rightarrow \mathbb{R}^{m}$ be a multifunction of closed graph around $(\bar{x}, \bar{y})$. Then one has

(2.22) $D^{*}(f+\Phi)(\bar{x}, f(\bar{x})+\bar{y})\left(y^{*}\right)=(\nabla f(\bar{x}))^{*} y^{*}+D^{*} \Phi(\bar{x}, \bar{y})\left(y^{*}\right) \quad \forall y^{*} \in \mathbb{R}^{m}$.

Proof. Let us put $\Phi_{1}=f$ and $\Phi_{2}=\Phi$ in Proposition 2.6. Then set (2.19) is obviously bounded and condition (2.20) is fulfilled due to Proposition 2.5(ii). Thus (2.21) implies the inclusion $\subset$ in (2.22). To prove the opposite inclusion $\supset$, we put $\Phi_{1}=-f$ and $\Phi_{2}=f+\Phi$ in Proposition 2.6 and use again Proposition 2.5(ii).

In this paper one can find many applications of Proposition 2.6 and its Corollaries 2.7 and 3.6. Note that the calculation of the coderivative (2.5) for singlevalued Lipschitzian vector functions can be reduced to the subdifferential (2.13) due to the following result proved in [9, Proposition 8 and 19, Corollary 3.3.2].

Proposition 2.8. Let $f: \mathbb{R}^{n} \rightarrow \mathbb{R}^{m}$ be a single-valued vector function Lipschitz continuous around $\bar{x}$ and let

$$
x \rightarrow\left\langle y^{*}, f\right\rangle(x):=\left\langle y^{*}, f(x)\right\rangle, \quad y^{*} \in \mathbb{R}^{m},
$$

be the Lagrange scalarization of $f$. Then one has

$$
D^{*} f(\bar{x})\left(y^{*}\right)=\partial^{-}\left\langle y^{*}, f\right\rangle(\bar{x}) \neq \varnothing \quad \forall y^{*} \in \mathbb{R}^{m} .
$$


Note that one cannot obtain an analogue of representation (2.24) stated in terms of the Clarke coderivative (2.6) and generalized gradient (2.16) in the nonsmooth case. Indeed, $D_{C}^{*} f(\bar{x})(0)=\{0\}$ if and only if $f$ is strictly differentiable at $\bar{x}$; see Proposition 3.1 and Corollary 3.2. On the other hand, Proposition 2.8 implies the following connection between the convex hull of the coderivative (2.5) and (Clarke's) generalized Jacobian $J f(\bar{x}) \subset \mathbb{R}^{m \times n}$ of Lipschitz continuous vector functions. Remind that $J f(\bar{x})$ is defined as the convex hull of the set of all limiting points for sequences $\left\{\nabla f\left(x_{k}\right)\right\}$ where $f$ is differentiable at $x_{k} \rightarrow \bar{x}$; see [4, p. 70].

Corollary 2.9. Let $f: \mathbb{R}^{n} \rightarrow \mathbb{R}^{m}$ be Lipschitz continuous around $\bar{x}$. Then one has

$$
\operatorname{co} D^{*} f(\bar{x})\left(y^{*}\right)=\operatorname{co} \partial^{-}\left\langle y^{*}, f\right\rangle(\bar{x})=(J f(\bar{x}))^{*} y^{*} \quad \forall y^{*} \in \mathbb{R}^{m} \text {. }
$$

Proof. This follows from (2.24), (2.16), and the chain rule in Clarke [4, Theorem 2.6.6].

Now let us consider a general chain rule for the subdifferential (2.13) of the composition $(\varphi \circ f)(x):=\varphi(f(x))$ of extended-real-valued and vector functions. For the formulation of the next result proved in Mordukhovich [19, Theorems 4.6 and 4.7], we need the following construction of the singular subdifferential of $\varphi: \mathbb{R}^{n} \rightarrow \overline{\mathbb{R}}$ at $\bar{x} \in \operatorname{dom} \varphi$.

$$
\begin{aligned}
\partial^{\infty} \varphi(\bar{x}) & :=D^{*} E_{\varphi}(\bar{x}, \varphi(\bar{x}))(0) \\
& =\left\{x^{*} \in \mathbb{R}^{n}:\left(x^{*}, 0\right) \in N((\bar{x}, \varphi(\bar{x})) \mid \operatorname{epi} \varphi)\right\} .
\end{aligned}
$$

Note that if $\varphi$ is 1.s.c. around $\bar{x}$, then $\partial^{\infty} \varphi(\bar{x})=\{0\}$ if and only if $\varphi$ is Lipschitz continuous around this point (see, e.g., [19, Theorem 2.1]).

Proposition 2.10. Let $f: \mathbb{R}^{n} \rightarrow \mathbb{R}^{m}$ be continuous around $\bar{x}, \varphi: \mathbb{R}^{m} \rightarrow \overline{\mathbb{R}}$ be l.s.c. around $\bar{y}=f(\bar{x}) \in \operatorname{dom} \varphi$, and let

$$
\partial^{\infty} \varphi(\bar{y}) \cap \operatorname{Ker} D^{*} f(\bar{x})=\{0\} .
$$

Then one has

$$
\partial^{-}(\varphi \circ f)(\bar{x}) \subset \bigcup\left[D^{*} f(\bar{x})\left(y^{*}\right): y^{*} \in \partial^{-} \varphi(\bar{y})\right] .
$$

Moreover, if $f$ is Lipschitz continuous around $\bar{x}$, then (2.28) becomes the equality

$$
\partial^{-}(\varphi \circ f)(\bar{x})=\bigcup\left[\partial^{-}\left\langle y^{*}, f\right\rangle(\bar{x}): y^{*} \in \partial^{-} \varphi(\bar{y})\right]
$$

if one of the following conditions holds:

(a) $f$ is strictly differentiable at $\bar{x}$ and $\varphi$ is subdifferentially regular at $\bar{y}$ which imply the subdifferential regularity of the composition $(\varphi \circ f)$;

(b) $f$ is strictly differentiable at $\bar{x}$ and the Jacobian matrix $\nabla f(\bar{x})$ is quadratic $(m=n)$ and nonsingular;

(c) $\varphi$ is strictly differentiable at $\bar{y}$.

Note that assumption (2.27) is automatically fulfilled if either $\varphi$ is locally Lipschitzian around $\bar{y}$ (as in (c)) or $f$ satisfies (b). Let us consider some corollaries of Proposition 2.10 which are important for applications in this paper. 
Corollary 2.11. Let

$$
\Delta:=\left\{x \in \mathbb{R}^{n}: f(x) \in \Lambda\right\}
$$

where the function $f: \mathbb{R}^{n} \rightarrow \mathbb{R}^{m}$ is continuous around $\bar{x}$, the set $\Lambda$ is closed around the point $f(\bar{x}) \in \Lambda$, and

$$
N(f(\bar{x}) \mid \Lambda) \cap \operatorname{Ker} D^{*} f(\bar{x})=\{0\} .
$$

Then one has the inclusion

$$
N(\bar{x} \mid \Delta) \subset \bigcup\left[D^{*} f(\bar{x})\left(y^{*}\right): y^{*} \in N(f(\bar{x}) \mid \Lambda)\right] .
$$

Moreover, if $f$ is strictly differentiable at $\bar{x}$, then (2.31) becomes an equality with $D^{*} f(\bar{x})\left(y^{*}\right)=(\nabla f(\bar{x}))^{*} y^{*}$ in the following two cases:

(a) the set $\Lambda$ is regular at $\bar{x}$ which implies the regularity of $\Delta$ at $f(\bar{x})$;

(b) the matrix $\nabla f(\bar{x})$ is quadratic and nonsingular which ensures (2.30).

Proof. Taking $\varphi=\delta(\cdot, \Lambda)$ in Proposition 2.10, one has $\partial^{-} \delta(f(\bar{x}), \Lambda)=$ $N(f(\bar{x} \mid \Lambda)$. Therefore, (2.27) is reduced to (2.30) and (2.28) coincides with (2.31). Equality conditions (a) and (b) in Proposition 2.10 and Corollary 2.11 are the same.

Corollary 2.12. Let the sets $\Omega_{1}, \Omega_{2} \subset \mathbb{R}^{n}$ be closed, let the function $f: \mathbb{R}^{n} \rightarrow$ $\mathbb{R}^{n}$ be strictly differentiable at $\bar{x} \in \Omega_{1}$ with the Jacobian matrix $\nabla f(\bar{x})$ being quadratic and nonsingular, and let

$$
\Omega_{1}=f^{-1}\left(\Omega_{2}\right) \text {. }
$$

Then one has

$$
N\left(\bar{x} \mid \Omega_{1}\right)=(\nabla f(\bar{x}))^{*} N\left(f(\bar{x}) \mid \Omega_{2}\right) .
$$

Proof. One can rewrite $(2.32)$ in the equivalent form

$$
\delta\left(x, \Omega_{1}\right)=\delta\left(f(x), \Omega_{2}\right)=\left(\delta\left(\cdot, \Omega_{2}\right) \circ f\right)(x) .
$$

Now using the equality case (b) in Proposition 2.10 for $\varphi=\delta\left(\cdot, \Omega_{2}\right)$, we obtain (2.33) which coincides with (2.29) by virtue of (2.18) and Proposition 2.5(ii).

To conclude this section, let us consider an extended-real-valued function $\varphi$ of two variables $(u, v) \in \mathbb{R}^{k} \times \mathbb{R}^{q}$. Proposition 2.10 allows us to obtain a general relationship between the "full' subdifferential $\partial^{-} \varphi$ in both variables and its "partial" subdifferentials with respect to $u$ and $v$. Remind that the partial subdifferentials $\partial_{u}^{-} \varphi(\bar{u}, \bar{v})$ and $\partial_{v}^{-} \varphi(\bar{u}, \bar{v})$ are defined, respectively, as the subdifferentials (2.13) of the functions $\varphi(\cdot, \bar{v})$ at $\bar{u}$ and the function $\varphi(\bar{u}, \cdot)$ at $\bar{v}$. Denote by $\operatorname{pr}_{u} \partial^{-} \varphi(\bar{u}, \bar{v})$ and $\operatorname{pr}_{v} \partial^{-} \varphi(\bar{u}, \bar{v})$ the corresponding projections of the set $\partial^{-} \varphi(\bar{u}, \bar{v})$ on $\mathbb{R}^{k}$ and $\mathbb{R}^{q}$.

Corollary 2.13. Let $\varphi: \mathbb{R}^{k} \times \mathbb{R}^{q} \rightarrow \overline{\mathbb{R}}$ be locally Lipschitzian around $(\bar{u}, \bar{v})$. Then one has

$$
\partial_{u}^{-} \varphi(\bar{u}, \bar{v}) \times \partial_{v}^{-} \varphi(\bar{u}, \bar{v}) \subset \operatorname{pr}_{u} \partial^{-} \varphi(\bar{u}, \bar{v}) \times \operatorname{pr}_{v} \partial^{-} \varphi(\bar{u}, \bar{v})
$$

where equality holds when $\varphi$ is separable in $(u, v)$, i.e., $\varphi(u, v)=\varphi_{1}(u)+$ $\varphi_{2}(v)$.

Proof. Let us use Proposition 2.10 with the Lipschitzian function $\varphi=\varphi(u, v)$ and the smooth function $f: \mathbb{R}^{k} \rightarrow \mathbb{R}^{k} \times \mathbb{R}^{q}$ defined by $f(u)=(u, \bar{v})$. From (2.28) one has

$$
\partial_{u}^{-} \varphi(\bar{u}, \bar{v}) \subset \operatorname{pr}_{u} \partial^{-} \varphi(\bar{u}, \bar{v})
$$


and similar inclusions for $\partial_{v}^{-} \varphi(\bar{u}, \bar{v})$. This implies (2.34). If $\varphi$ is separable in $(u, v)$, then

$$
\partial^{-} \varphi(\bar{u}, \bar{v})=\left(\partial^{-} \varphi_{1}(\bar{u}), \partial^{-} \varphi_{2}(\bar{v})\right)=\left(\partial_{u}^{-} \varphi(\bar{u}, \bar{v}), \partial_{v}^{-} \varphi(\bar{u}, \bar{v})\right)
$$

which ensures equality in (2.34).

From the result obtained and Corollary 2.9, one can easily derive the following relationship between the generalized Jacobian $J f$ of any Lipschitzian vector function $f=f(u, v): \mathbb{R}^{k} \times \mathbb{R}^{q} \rightarrow \mathbb{R}^{m}$ and the partial Jacobians $J_{u} f$ and $J_{v} f$. Denote by $\operatorname{pr}_{u} J f(\bar{u}, \bar{v})$ and $\operatorname{pr}_{v} J f(\bar{u}, \bar{v})$ the projections of the set $J f(\bar{u}, \bar{v}) \subset \mathbb{R}^{m k} \times \mathbb{R}^{m q}$ on the spaces $\mathbb{R}^{m k}$ and $\mathbb{R}^{m q}$ respectively.

Corollary 2.14. Let $f: \mathbb{R}^{k} \times \mathbb{R}^{q} \rightarrow \mathbb{R}^{m}$ be Lipschitz continuous around $(\bar{u}, \bar{v})$. Then one has

$$
\left(J_{u} f(\bar{u}, \bar{v})\right)^{*} y^{*} \subset\left(\operatorname{pr}_{u} J f(\bar{u}, \bar{v})\right)^{*} y^{*} \quad \forall y^{*} \in \mathbb{R}^{m}
$$

and the same inclusion with respect to $v$. Both inclusions are fulfilled as equalities if $f$ is separable in $(u, v)$.

Proof. For any $y^{*} \in \mathbb{R}^{m}$, let us consider the real-valued function $\varphi(u, v):=$ $\left\langle y^{*}, f(u, v)\right\rangle$ and let us use the result in Corollary 2.13 for this function. Employing (2.34), Corollary 2.9, and two elementary relationships, one has

$$
\begin{aligned}
\left(J_{u} f(\bar{u}, \bar{v})\right)^{*} y^{*} & =\operatorname{co} \partial_{u}^{-}\left\langle y^{*}, f\right\rangle(\bar{u}, \bar{v}) \subset \operatorname{co}\left[\operatorname{pr}_{u}\left\langle y^{*}, f\right\rangle(\bar{u}, \bar{v})\right] \\
& \subset \operatorname{pr}_{u}\left[\operatorname{co} \partial^{-}\left\langle y^{*}, f\right\rangle(\bar{u}, \bar{v})\right]=\operatorname{pr}_{u}\left[(J f(\bar{u}, \bar{v}))^{*} y^{*}\right] \\
& =\left(\operatorname{pr}_{u} J f(\bar{u}, \bar{v})\right)^{*} y^{*} \quad \forall y^{*} \in \mathbb{R}^{m}
\end{aligned}
$$

which proves $(2.35)$. The equality case in the assertion proved is obvious.

\section{SMOOTH AND LIPSCHITZIAN PROPERTIES OF MULTIFUNCTIONS}

In this section we consider some results about smooth and Lipschitzian properties of general multifunctions important in what follows. We begin with the concepts of Lipschitzian and strictly smooth manifolds which are slight modifications of Rockafellar's concepts in [35].

A set $S \subset \mathbb{R}^{q}$ is said to be a Lipschitzian manifold around the point $\bar{s} \in S$ if it is locally representable as the graph of a Lipschitz continuous vector function in the following sense: there exist an open neighborhood $W$ of $\bar{s}$ and a one-toone mapping $f$ of $W$ onto an open set in $\mathbb{R}^{n} \times \mathbb{R}^{m}$ (where $n+m=q$ ) such that $f$ is strictly differentiable at $\bar{s}$ with the nonsingular Jacobian $\nabla f(\bar{s})$ and $f(S \cap W)$ is the graph of some Lipschitzian function $g: U \rightarrow \mathbb{R}^{m}$ where $U$ is an open set in $\mathbb{R}^{n}$. One can see that the integer $n$ in this definition is uniquely determined by $S$ and $\bar{s}$; this number is called the dimension of $S$ around $\bar{s}$.

It is obvious that the graph of any function $g: \mathbb{R}^{n} \rightarrow \mathbb{R}^{m}$ locally Lipschitzian around $\bar{x}$ is a Lipschitzian manifold of dimension $n$ around $(\bar{x}, g(\bar{x}))$. A less obvious example, which is of great interest for applications, is provided by graphs of maximal monotone multifunctions. Recall that the operator $\Phi: \mathbb{R}^{n} \Rightarrow$ $\mathbb{R}^{n}$ is called positive monotone if

$$
\left\langle x_{1}-x_{2}, y_{1}-y_{2}\right\rangle \geq 0 \quad \forall x_{1}, x_{2} \text { and } y_{1} \in \Phi\left(x_{1}\right), y_{2} \in \Phi\left(x_{2}\right) \text {. }
$$

If one changes the sign of inequality (3.1), then $\Phi$ is called negative monotone. We say that $\Phi$ is monotone if it is either positive or negative monotone. A 
monotone operator $\Phi: \mathbb{R}^{n} \Rightarrow \mathbb{R}^{n}$ is called maximal monotone if its graph includes graphs of any other monotone operators from $\mathbb{R}^{n}$ into itself in the same direction. The class of maximal monotone operators contains subdifferential (superdifferential) mappings for convex, concave, and saddle functions; see [32, $\S \S 24,35]$ for more details.

It has been proved in Rockafellar [35, Proposition 2.2] that if $\Phi: \mathbb{R}^{n} \Rightarrow \mathbb{R}^{n}$ is maximal monotone, then its graph is a Lipschitzian manifold of dimension $n$ in $\mathbb{R}^{n} \times \mathbb{R}^{n}$ around any point $(\bar{x}, \bar{y}) \in \operatorname{gph} \Phi$. This implies, in particular, that graphs of subdifferential operators for convex, concave, and saddle functions are Lipschitzian manifolds. Note also that the class of Lipschitzian manifolds includes graphs of subdifferential mappings for the strongly subsmooth (lower$\mathbb{C}^{2}$ ) functions which can be locally represented as a maximum of $C^{2}$ functions over compact sets; see [35, §2].

A Lipschitzian manifold $S$ is called strictly smooth at $\bar{s} \in S$ if a Lipschitz continuous function $g$ in its local representation around $\bar{s}$ can be chosen as strictly differentiable at the point $\bar{x}$ with $(\bar{x}, g(\bar{x}))=f(\bar{s})$. We refer to Rockafellar [35] for equivalent characterizations and more details about strictly smooth manifolds. The next result follows from [35, Theorem 3.5] due to simple considerations of linear algebra.

Proposition 3.1. Let $\Phi$ be a multifunction from $\mathbb{R}^{n}$ into $\mathbb{R}^{m}$ whose graph is a Lipschitzian manifold of some dimension $q \geq n$ around $(\bar{x}, \bar{y}) \in \operatorname{gph} \Phi$. Then the graph of $\Phi$ is strictly smooth at $(\bar{x}, \bar{y})$ if and only if the Clarke normal cone to $\operatorname{gph} \Phi$ is actually a linear subspace of dimension $q$. It always happens with $q=n$ if

$$
D_{C}^{*} \Phi(\bar{x}, \bar{y})(0)=\{0\} .
$$

Moreover, if $\operatorname{gph} \Phi$ is not strictly smooth at $(\bar{x}, \bar{y})$, then for any $y^{*} \in \mathbb{R}^{m}$ the set $D_{C}^{*} \Phi(\bar{x}, \bar{y})\left(y^{*}\right)$ is either empty or it is an affine subspace in $\mathbb{R}^{n}$ of a positive dimension.

Proof. Let us consider the Clarke normal cone (2.2) to the graph of $\Phi$ at $(\bar{x}, \bar{y})$. Using Theorem 3.5(b) in Rockafellar [35], one can conclude that this cone is actually a linear subspace of dimension $d \geq q$ in $\mathbb{R}^{n} \times \mathbb{R}^{m}$. Therefore, taking into account the definition of the Clarke coderivative (2.6), we can find matrices $K \in \mathbb{R}^{d \times n}$ and $L \in \mathbb{R}^{d \times m}$ such that the extended matrix $(K L) \in \mathbb{R}^{n+m}$ has the full rank $d \leq n+m$ and

$$
D_{C}^{*} \Phi(\bar{x}, \bar{y})\left(y^{*}\right)=\left\{x^{*} \in \mathbb{R}^{n}: K x^{*}=L y^{*}\right\} \quad \forall y^{*} \in \mathbb{R}^{m} .
$$

According to [35, Theorem 3.5(c)], the graph of $\Phi$ is a strictly smooth manifold at $(\bar{x}, \bar{y})$ if and only if the dimension $d$ of the Clarke normal cone above is exactly $q$. Assuming (3.2), one immediately has $d=n=q$ and, therefore, the graph of $\Phi$ is strictly smooth at $(\bar{x}, \bar{y})$. If the graph of $\Phi$ is not strictly smooth at $(\bar{x}, \bar{y})$, then $\operatorname{rank}(K)=k \leq n<d$ and the dimension of set (3.3) is equal to $d-k \geq 1$ for any $y^{*} \in \operatorname{Dom} D_{C}^{*} \Phi(\bar{x}, \bar{y})$.

Note that condition (3.2) is sufficient but not necessary for the graph of $\Phi$ being strictly smooth at $(\bar{x}, \bar{y})$. A simple counterexample is provided by the graph of the real-valued function $x^{1 / 3}$ at $(0,0) \in \mathbb{R}^{2}$. 
Corollary 3.2. Let $g: \mathbb{R}^{n} \rightarrow \mathbb{R}^{m}$ be a single-valued function locally Lipschitzian around $\bar{x}$. Then $g$ is differentially regular at $\bar{x}$ if and only if it is strictly differentiable at this point.

Proof. If $g$ is strictly differentiable at $\bar{x}$, then its differential regularity at this point follows from Proposition 2.5(ii). If $g$ is not strictly differentiable at $\bar{x}$, then according to Proposition 3.1, the set $D_{C}^{*} g(\bar{x})(0)$ is a linear subspace of a positive dimension. On the other hand, one has

$$
D^{*} g(\bar{x})(0)=\partial^{-}\langle 0, g\rangle(\bar{x})=\{0\}
$$

by virtue of Proposition 2.8 .

Based on the result in Corollary 3.2, we can obtain its extension to the case of multifunctions with Lipschitzian graphs employing a calculus rule for the normal cones.

Proposition 3.3. Let $\Phi: \mathbb{R}^{n} \Rightarrow \mathbb{R}^{m}$ be a multifunction whose graph is a Lipschitzian manifold around $(\bar{x}, \bar{y}) \in \operatorname{gph} \Phi$. Then $\Phi$ is differentially regular at $(\bar{x}, \bar{y})$ if and only if its graph is strictly smooth at this point.

Proof. Because of the graph of $\Phi$ being a Lipschitzian manifold around $(\bar{x}, \bar{y})$, one can find a function $f: \mathbb{R}^{n+m} \rightarrow \mathbb{R}^{n+m}$ strictly differentiable at $(\bar{x}, \bar{y})$ with

$$
\operatorname{det}(\nabla f(\bar{x}, \bar{y}))=n+m
$$

and a function $g: \mathbb{R}^{n} \rightarrow \mathbb{R}^{m}$ Lipschitz continuous around the point $\bar{u} \in \mathbb{R}^{n}$ with $(\bar{u}, g(\bar{u}))=f(\bar{x}, \bar{y})$ such that

$$
\operatorname{gph} \Phi=f^{-1}(\operatorname{gph} g)
$$

in some neighborhood of $(\bar{x}, \bar{y})$, where $f^{-1}$ exists due to the classical inverse function theorem. Now considering the normal cones (2.1) to both sets in (3.4) and employing Corollary 2.12, we obtain the equality

$$
N((\bar{x}, \bar{y}) \mid \operatorname{gph} \Phi)=(\nabla f(\bar{x}, \bar{y}))^{*} N((\bar{u}, g(\bar{u})) \mid \operatorname{gph} g) .
$$

Taking the convex closure to both parts in (3.5), one has the same equality for the Clarke normal cones (2.2) to sets (3.4). It follows from (2.5) and (2.6) that $\Phi$ is differentially regular at $(\bar{x}, \bar{y})$ if and only if

$$
N((\bar{x}, \bar{y}) \mid \operatorname{gph} \Phi)=N_{C}((\bar{x}, \bar{y}) \mid \operatorname{gph} \Phi) .
$$

By virtue of (3.5), the latter is equivalent to

$$
N((\bar{u}, g(\bar{u})) \mid \operatorname{gph} g)=N_{C}((\bar{u}, g(\bar{u})) \mid \operatorname{gph} g)
$$

which means that the function $g$ is differentially regular at $\bar{u}$. Therefore, the general case in Proposition 3.3 is reduced to the single-valued case in Corollary 3.2.

The results obtained show that the coderivatives (2.5) and (2.6) are dramatically different (in dimensions!) for any multifunctions whose graphs are Lipschitzian manifolds around points under consideration. This is related to the fact that condition (3.2) implies the strict smooth property considered above, while its analogue in terms of the coderivative (2.6) completely characterizes the following Lipschitzian behavior of multifunctions. 
The multifunction $\Phi: \mathbb{R}^{n} \Rightarrow \mathbb{R}^{m}$ with the closed graph is said to be pseudoLipschitzian around $(\bar{x}, \bar{y}) \in \operatorname{gph} \Phi$ if there exist a neighborhood $U$ of $\bar{x}$, a neighborhood $V$ of $\bar{y}$, and a constant $l \geq 0$ such that

$$
\Phi\left(x^{\prime}\right) \cap V \subset \Phi(x)+l\left\|x^{\prime}-x\right\| B \text { for any } x, x^{\prime} \in U
$$

where $B \subset \mathbb{R}^{n}$ is the unit closed ball. Observe that the pseudo-Lipschitzian property of $\Phi$ always implies the nonemptiness of the sets $\Phi(x)$ around $\bar{x}$; this follows from (3.6) when $x^{\prime}=\bar{x}$.

If for every compact set $V \subset \mathbb{R}^{m}$ there exist a neighborhood $U$ of $\bar{x}$ and a number $l \geq 0$ such that (3.6) holds, then the multifunction $\Phi$ is called subLipschitzian around $\bar{x} \in \operatorname{Dom} \Phi$. Finally, the multifunction $\Phi$ is called locally Lipschitzian around $\bar{x} \in \operatorname{Dom} \Phi$ if there exist a neighborhood $U$ of $\bar{x}$ and a number $l \geq 0$ such that (3.6) is fulfilled with $V=\mathbb{R}^{m}$.

Observe that the latter locally Lipschitzian property coincides with the notion of Lipschitzness for multifunctions $\Phi: \mathbb{R}^{n} \Rightarrow \mathbb{R}^{m}$ treated as single-valued mappings from $\mathbb{R}^{n}$ into the space of all nonempty compact subsets of $\mathbb{R}^{m}$ with the Hausdorff metric on this space. Note also that both properties of pseudoLipschitzness, introduced by Aubin [1], and sub-Lipschitzness, introduced by Rockafellar [34], extend the locally (around $\bar{x}$ ) Lipschitzian behavior to the case of unbounded multifunctions. If $\Phi$ is single-valued around $\bar{x}$, then all three concepts under consideration are reduced to the classical locally Lipschitz continuity of vector functions.

Recall the multifunction $\Phi$ is said to be locally bounded around $\bar{x}$ if there is a neighborhood $U$ of $\bar{x}$ such that the set $\Phi(U)$ is bounded. The following assertions can be found in Rockafellar [34, Theorems 2.1 and 2.2].

Proposition 3.4. For any closed-graph multifunction $\Phi$ one has:

(i) $\Phi$ is sub-Lipschitzian around $\bar{x}$ if and only if $\Phi$ is pseudo-Lipschitzian around $(\bar{x}, \bar{y})$ for every point $\bar{y} \in \Phi(\bar{x})$;

(ii) $\Phi$ is locally Lipschitzian around $\bar{x}$ if and only if $\Phi$ is locally bounded and sub-Lipschitzian around this point.

Let us formulate a criterion for the pseudo-Lipschitzian property of multifunctions on which main results in this paper are based.

Proposition 3.5. Let $\Phi: \mathbb{R}^{n} \Rightarrow \mathbb{R}^{m}$ be a multifunction with the closed graph. Then $\Phi$ is pseudo-Lipschitzian around $(\bar{x}, \bar{y})$ if and only if

$$
D^{*} \Phi(\bar{x}, \bar{y})=\{0\} .
$$

This result is proved in Mordukhovich [20, Theorem 5.4; 22, Theorem 5.7] by different ways. In [22, 23], one can find some other criteria for $\Phi$ being pseudoLipschitzian around $(\bar{x}, \bar{y})$ and also precise formulae for evaluating the exact bound of Lipschitz moduli $l$ in (3.6) expressed in terms of the coderivative (2.5). Using criterion (3.7), we obtain the following effective realization of the calculus rule in Proposition 2.6.

Corollary 3.6. Let $f: \mathbb{R}^{n} \rightarrow \mathbb{R}^{m}$ be a vector function continuous around $\bar{x}$ and let $\Phi: \mathbb{R}^{n} \Rightarrow \mathbb{R}^{m}$ be a multifunction of closed graph around $(\bar{x}, \bar{y}) \in \operatorname{gph} \Phi$. Then one has

$$
D^{*}(f+\Phi)(\bar{x}, f(\bar{x})+\bar{y})\left(y^{*}\right) \subset D^{*} f(\bar{x})\left(y y^{*}\right)+D^{*} \Phi(\bar{x}, \bar{y})\left(y^{*}\right) \quad \forall y^{*} \in \mathbb{R}^{m}
$$


if either $f$ is Lipschitz continuous around $\bar{x}$ or $\Phi$ is pseudo-Lipschitzian around $(\bar{x}, \bar{y})$.

Proof. This follows from Propositions 2.6 and 3.5.

The next criteria for the sub-Lipschitzian and locally Lipschitzian properties of multifunctions follow directly from Propositions 3.4 and 3.5.

Corollary 3.7. For any closed-graph multifunction $\Phi$ being sub-Lipschitzian around $\bar{x}$, it is necessary and sufficient that

$$
D^{*} \Phi(\bar{x}, \bar{y})(0)=\{0\} \quad \text { for every } \bar{y} \in \Phi(\bar{x}) .
$$

If the multifunction $\Phi$ is locally bounded around $\bar{x}$, then condition (3.8) is necessary and sufficient for $\Phi$ being locally Lipschitzian around this point.

Now employing Proposition 3.4 and Corollary 3.7 to the inverse multifunction

$$
\Phi^{-1}(y):=\left\{x \in \mathbb{R}^{n}:(x, y) \in \operatorname{gph} \Phi\right\},
$$

one has the following characterizations.

Corollary 3.8. Let $\Phi$ be a closed-graph multifunction and let $(\bar{x}, \bar{y}) \in \operatorname{gph} \Phi$. Then:

(i) For the inverse (3.9) to be pseudo-Lipschitzian around $(\bar{y}, \bar{x})$, it is necessary and sufficient that

$$
\operatorname{Ker} D^{*} \Phi(\bar{x}, \bar{y})=\{0\} .
$$

(ii) For the inverse (3.9) to be sub-Lipschitzian around $\bar{y}$, it is necessary and sufficient that

$$
\operatorname{Ker} D^{*}(x, \bar{y})=\{0\} \quad \text { for every } x \in \Phi^{-1}(\bar{y}) \text {. }
$$

(iii) Let, in addition, $\Phi^{-1}$ be locally bounded around $\bar{y}$. Then condition (3.11) is necessary and sufficient for multifunction (3.9) being locally Lipschitzian around this point.

Proof. This follows from Propositions 3.4 and 3.5 due to the relationship

$$
y^{*} \in D^{*} \Phi^{-1}(\bar{y}, \bar{x})\left(x^{*}\right) \Leftrightarrow-x^{*} \in D^{*} \Phi(\bar{x}, \bar{y})\left(-y^{*}\right) .
$$

Remark 3.9. If one changes criterion (3.10) to

$$
\operatorname{Ker} D_{C}^{*} \Phi(\bar{x}, \bar{y})=\{0\}
$$

in terms of the Clarke coderivative (2.6), then the condition obtained is sufficient for the pseudo-Lipschitzian property of the inverse mapping $\Phi^{-1}$ around $(\bar{y}, \bar{x})$. This version of the inverse mapping theorem has been first proved by Aubin [1, §3] and Rockafellar [34, §3]. Employing Proposition 3.1, we can observe that if the graph of $\Phi$ is a Lipschitzian manifold of dimension $n$ around $(\bar{x}, \bar{y})$, then condition (3.12) is far removed from the necessity of $\Phi^{-1}$ being pseudo-Lipschitzian: it actually implies not only the pseudo-Lipschitzian but also smoothness property of the inverse mapping.

Remark 3.10. It is well known that the pseudo-Lipschitzian property of the inverse operator (3.9) is equivalent to the metric regularity and openness (at a linear rate) property of the multifunction $\Phi$ itself; see $[3,22,25]$. Therefore, 
(3.10) provides a criterion for the mentioned properties of any closed-graph multifunction. We refer to the article [22] for more details about this and related topics.

The next useful result about the pseudo-Lipschitzian property of the composition of two Lipschitzian multifunctions follows from Rockafellar [34, Theorem 4.1].

Proposition 3.11. Let the multifunction $\Phi: \mathbb{R}^{n} \Rightarrow \mathbb{R}^{m}$ be given by

$$
\Phi(x)=\Phi_{1}\left(\Phi_{0}(x)\right):=\bigcup\left[\Phi_{1}(u): u \in \Phi_{0}(x)\right]
$$

where $\Phi_{0}: \mathbb{R}^{n} \Rightarrow \mathbb{R}^{k}$ and $\Phi_{1}: \mathbb{R}^{k} \Rightarrow \mathbb{R}^{m}$ are multifunctions of closed graph. For given $(\bar{x}, \bar{y}) \in \operatorname{gph} \Phi$, we assume that $\Phi_{0}$ is locally Lipschitzian around $\bar{x}$ and $\Phi_{1}$ is pseudo-Lipschitzian around any $(u, \bar{y})$ with $u \in \Phi_{0}(\bar{x}) \cap \Phi_{1}^{-1}(\bar{y})$. Then the graph of $\Phi$ is closed and $\Phi$ is pseudo-Lipschitzian around $(\bar{x}, \bar{y})$.

Now let us consider again a class of monotone operators $\Phi: \mathbb{R}^{n} \Rightarrow \mathbb{R}^{n}$. As we mentioned above, the graph of any such $\Phi$ is a Lipschitzian manifold around any point $(\bar{x}, \bar{y}) \in \operatorname{gph} \Phi$. Moreover, it turns out that if $\Phi$ is pseudoLipschitzian around $(\bar{x}, \bar{y})$, then this multifunction is actually single-valued around $\bar{x}$. The proof of the following assertion can be found in Dontchev and Hager [6, Proposition 5.1].

Proposition 3.12. Let $\Phi$ be a monotone multifunction which is pseudo-Lipschitzian around $(\bar{x}, \bar{y})$. Then $\Phi$ is single-valued (and Lipschitz continuous) in a neighborhood of $\bar{x}$.

Consider some applications of results obtained in Propositions 3.1, 3.5, and 3.12 to the case subdifferential mappings for convex functions.

Corollary 3.13. Let $\varphi: \mathbb{R}^{n} \rightarrow \overline{\mathbb{R}}$ be a closed proper convex function with the subdifferential $\partial \varphi$ in the sense of convex analysis and let $\partial^{2,-} \varphi(\bar{x}, \bar{y})$ be its second order subdifferential (2.14) at $\bar{x} \in \operatorname{dom} \varphi$ relative to some $\bar{y} \in \partial \varphi(\bar{x})$. Then the condition

$$
\partial^{2,-} \varphi(\bar{x}, \bar{y})(0)=\{0\}
$$

is necessary and sufficient for the function $\varphi$ being continuously differentiable in some neighborhood of $\bar{x}$ with the gradient mapping $\nabla \varphi: \mathbb{R}^{n} \rightarrow \mathbb{R}^{n}$ being Lipschitz continuous around $\bar{x}$.

Proof. According to definition (2.14), the second order condition (3.13) is equivalent to (3.7) for the subdifferential multifunction $\Phi=\partial \varphi$. Using Proposition 3.5 , we conclude that (3.13) is a criterion for $\partial \varphi$ being pseudo-Lipschitzian around $(\bar{x}, \bar{y})$. Now employing Corollary 3.13 , one can see that (3.13) is actually a criterion for the single-valuedness and Lipschitz continuity of $\partial \varphi$ in some neighborhood of $\bar{x}$ where $\partial \varphi \equiv \nabla \varphi$.

Corollary 3.14. Let $\varphi: \mathbb{R}^{n} \rightarrow \overline{\mathbb{R}}$ be a closed proper convex function and let $\partial_{C}^{2,-} \varphi(\bar{x}, \bar{y})$ be its Clarke second order subdifferential (2.17). Then the condition

$$
\partial_{C}^{2,-} \varphi(\bar{x}, \bar{y})(0)=\{0\}
$$


implies that there exists a neighborhood of $\bar{x}$ on which $\varphi$ enjoys the following (classical) twice differentiability property: $\varphi$ is continuously differentiable and its gradient mapping $\nabla \varphi$ is strictly differentiable at $\bar{x}$.

Proof. Condition (3.14) implies (3.13) and, due to the previous assertion, it ensures that $\varphi$ is continuously differentiable around $\bar{x}$. On the other hand, (3.14) coincides with (3.2) for the multifunction $\Phi=\partial \varphi$. By virtue of Proposition 3.1 , this condition implies that the gradient mapping $\nabla \varphi \equiv \partial \varphi$ is strictly differentiable at $\bar{x}$ which means the twice differentiability property of the function $\varphi$.

Remark 3.15. Condition (3.14) ensures that the Clarke normal cone to the graph of $\partial \varphi$ at $(\bar{x}, \bar{y})$ is a linear subspace of dimension $n$ in $\mathbb{R}^{2 n}$. The latter is in general equivalent to the graph of $\partial \varphi$ being strictly smooth at $(\bar{x}, \bar{y})$; see Proposition 3.1. According to Rockafellar [35, $\S 4$, this property always implies that $\varphi$ must be in fact twice differentiable in the sense of having a second order Taylor expansion at the point in question. The observation made excludes the possibility of using the Clarke normal cone in sensitivity analysis of variational inequalities and complementarity problems where $\varphi$ is the indicator function of a convex set; see [34, Remark 3.13] and Remark 4.9 for more details.

\section{LINEAR GENERALIZED EQUATIONS}

We begin this section with studying linear perturbed generalized equations of the form

$$
0 \in A z+p+Q(z)
$$

where $z \in \mathbb{R}^{n}$ is a solution vector (decision variable), $p \in \mathbb{R}^{m}$ is a parameter, $A \in \mathbb{R}^{m \times n}$ is a fixed constant matrix, and $Q: \mathbb{R}^{n} \Rightarrow \mathbb{R}^{m}$ is an arbitrary multifunction of closed graph containing the point $(0,0)$. Such linear generalized equations have some important applications (in particular, they arise naturally from optimality conditions for quadratic programming; see, e.g., [30]). Moreover, they can be treated as an appropriate approximation (linearization) of nonlinear generalized equations in a broad setting; see $\S 5$. In this way, the results obtained for linear generalized equations play a basic role for studying nonlinear generalized equations via such an approximation.

Let us consider the set of solutions

$$
\Gamma(p):=\left\{z \in \mathbb{R}^{n}: 0 \in A z+p+Q(z)\right\}
$$

to the generalized equation (4.1) and let us study the dependence of the solution map $\Gamma$ on the parameter $p$. We are going to concentrate on the pseudoLipschitzian property of $\Gamma$ around $(0,0)$ which ensures, in particular, that sets (4.2) are nonempty for all $p$ around 0.

Now we obtain a necessary and sufficient condition for the multifunction $\Gamma: \mathbb{R}^{m} \Rightarrow \mathbb{R}^{n}$ in $(4.2)$ being pseudo-Lipschitzian around $(0,0)$. For this purpose, let us introduce the adjoint generalized equation

$$
0 \in A^{*} y^{*}+D^{*} Q(0,0)\left(y^{*}\right) \text { for } y^{*} \in \mathbb{R}^{m}
$$

to $(4.1)$ at $(0,0)$ in terms of the adjoint (transposed) matrix $A^{*}$ to $A$ and the coderivative (adjoint mapping) $(2.5)$ to $Q$ at $(0,0)$. We say that the adjoint equation (4.3) has only the trivial solution if

$$
\left[0 \in A^{*} y^{*}+D^{*} Q(0,0)\left(y^{*}\right)\right] \Rightarrow y^{*}=0 .
$$


Theorem 4.1. For the solution map (4.2) to the linear generalized equation (4.1) being pseudo-Lipschitzian around $(0,0)$, it is necessary and sufficient that the adjoint generalized equation (4.2) have only the trivial solution.

Proof. Let us consider the multivalued operator

$$
L(z):=A z+Q(z)
$$

and its inverse $L^{-1}: \mathbb{R}^{m} \Rightarrow \mathbb{R}^{n}$. It is obvious that the values of the inverse multifunction to (4.5) can be represented in the form

$$
L^{-1}(y)=\left\{z \in \mathbb{R}^{n}: 0 \in A z-y+Q(z)\right\},
$$

i.e., $L^{-1}(-p)$ coincides with $\Gamma(p)$ in (4.2). Therefore, the pseudo-Lipschitzness of the solution map (4.2) around $(0,0)$ is equivalent to the multifunction $L$ in (4.5) having the pseudo-Lipschitzian inverse around this point. According to Corollary 3.8(i), the latter property holds if and only if

$$
\left[0 \in D^{*} L(0,0)\left(y^{*}\right)\right] \Rightarrow y^{*}=0 .
$$

Using Corollary 2.7 for the coderivative of the sum in (4.5), one can conclude that (4.6) is equivalent to (4.4). This ends the proof of the theorem.

Now let us obtain effective representations of the Lipschitz stability criterion in Theorem 4.1 for some special classes of multifunctions $Q$ in the perturbed generalized equation (4.1).

Corollary 4.2. Let the multifunction $Q$ in (4.1) have the convex graph. Then the solution map (4.2) is pseudo-Lipschitzian around $(0,0)$ if and only if

$$
0 \in \operatorname{int}(\operatorname{Im} L)
$$

for the operator $L$ in (4.5).

Proof. Using Proposition 2.5(i) for the representation of the coderivative $D^{*} Q(0,0)\left(y^{*}\right)$ of the convex-graph multifunction $Q$, one can reduce (4.4) to

$$
\left[\left\langle A^{*} y^{*}, z\right\rangle+\left\langle y^{*}, y\right\rangle \geq 0 \forall(z, y) \in \operatorname{gph} Q\right] \Rightarrow y^{*}=0 .
$$

Let us consider the expression in the brackets of (4.8). Because $\left\langle A^{*} y^{*}, z\right\rangle=$ $\left\langle y^{*}, A z\right\rangle$, this expression means that $u=0$ is an optimal solution to the convex minimization problem

$$
\text { minimize }\left\langle y^{*}, u\right\rangle \text { subject to } u \in \operatorname{Im} L \text {. }
$$

Using a well-known necessary and sufficient optimality condition for this problem, we can see that criterion (4.8) is equivalent to

$$
\left[-y^{*} \in N(0 \mid \operatorname{Im} L)\right] \Rightarrow y^{*}=0 .
$$

The latter holds if and only if condition (4.7) is fulfilled.

Note that (4.7) is the interiority condition in the Robinson-Ursescu theorem for the operator $L$ in $(4.5)$; cf. [2, $\S 2.2]$. Let us consider a special case of Corollary 4.2 when

$$
Q(z)= \begin{cases}E & \text { for } z \in \Omega, \\ \varnothing & \text { otherwise }\end{cases}
$$

with $E \subset \mathbb{R}^{m}$ and $\Omega \subset \mathbb{R}^{n}$. Denote $A \Omega:=\left\{y \in \mathbb{R}^{m}: y=A z\right.$ for $\left.z \in \Omega\right\}$. 
Corollary 4.3. Let the multifunction $Q$ in (4.1) have form (4.9) where the sets $E$ and $\Omega$ are closed and convex. Then the solution map (4.2) to (4.1), (4.9) is pseudo-Lipschitzian around $(0,0)$ if and only if

$$
0 \in \operatorname{int}(A \Omega+E) \text {. }
$$

Proof. One can see that the multifunction $Q$ in (4.9) has convex graph and condition (4.10) is equivalent to (4.7) for this $Q$. Therefore, Corollary 4.3 follows directly from Corollary 4.2.

Now let us consider the case when the multifunction $Q$ in (4.1) is represented in the form

$$
Q(z):=\left\{y \in \mathbb{R}^{m}: \theta(z, y) \in \Lambda\right\}
$$

where $\Lambda \subset \mathbb{R}^{q}$ is a closed set and where $\theta: \mathbb{R}^{n} \times \mathbb{R}^{m} \rightarrow \mathbb{R}^{q}$ is an arbitrary vector function with $\theta(0,0) \in \Lambda$.

Corollary 4.4. Let the function $\theta$ in $(4.11)$ be continuous around $(0,0)$. Then the condition

$$
\begin{gathered}
{\left[\left(A^{*} y^{*}, y^{*}\right) \in D^{*} \theta(0,0)\left(w^{*}\right) \& w^{*} \in N(\theta(0,0) \mid \Lambda)\right]} \\
\Rightarrow w^{*}=0 \& y^{*}=0
\end{gathered}
$$

is sufficient for the solution map (4.2) to (4.1), (4.11) being pseudo-Lipschitzian around $(0,0)$. Moreover, if $\theta$ is strictly differentiable at $(0,0)$, then the condition

$$
\begin{aligned}
& {\left[\left(\nabla_{y} \theta(0,0) A-\nabla_{z} \theta(0,0)\right)^{*} w^{*}=0 \& w^{*} \in N(\theta(0,0) \mid \Lambda)\right]} \\
& \quad \Rightarrow\left(\nabla_{y} \theta(0,0)\right)^{*} w^{*}=0
\end{aligned}
$$

is necessary and sufficient for (4.2) being pseudo-Lipschitzian around $(0,0)$ when either

(a) $\nabla \theta(0,0)$ is quadratic $(q=n+m)$ and nonsingular, or

(b) $\Lambda$ is regular at $\theta(0,0)$ and

$$
\operatorname{Ker}(\nabla \theta(0,0))^{*} \cap N(\theta(0,0) \mid \Lambda)=\{0\} .
$$

Proof. According to the definition of the coderivative, one can represent criterion (4.4) for multifunction (4.11) in the form

$$
\left[\left(A^{*} y^{*}, y^{*}\right) \in N((0,0) \mid \Delta)\right] \Rightarrow y^{*}=0
$$

where $\Delta:=\left\{(z, y) \in \mathbb{R}^{n+m}: \theta(z, y) \in \Lambda\right\}$. Let us obtain a representation of the normal cone in (4.15) using Corollary 2.11. Due to this result, one has that criterion (4.15) is fulfilled if the following two conditions hold:

$$
\begin{gathered}
{\left[\left(A^{*} y^{*}\right) \in D^{*} \theta(0,0)\left(w^{*}\right) \& w^{*} \in N(\theta(0,0) \mid \Lambda)\right] \Rightarrow y^{*},} \\
\operatorname{Ker} D^{*} \theta(0,0) \cap N(\theta(0,0) \mid \Lambda)=\{0\} .
\end{gathered}
$$

Moreover, if $\theta$ is strictly differentiable at $(0,0)$ and $(4.14)$ is fulfilled, then, according to Corollary 2.11, criterion (4.15) is equivalent to (4.13) in cases (a) or (b) of the assertion being proved. To finish the proof of this result, we observe that (4.12) holds in the general nonsmooth setting if and only if (4.16) and (4.17) are fulfilled simultaneously.

Corollary 4.5. Let $\theta$ be Lipschitz continuous around $(0,0)$. Then the condition

$$
\left[\left(A^{*} y^{*}, y^{*}\right) \in \partial^{-}\left\langle w^{*}, \theta\right\rangle(0,0) \& w^{*} \in N(\theta(0,0) \mid \Lambda)\right] \Rightarrow w^{*}=0
$$


ensures the pseudo-Lipschitzness of the solution map (4.2) to (4.1), around $(0,0)$. This condition is automatically fulfilled if

$$
\left[\left(A^{*} y^{*}, y^{*}\right) \in(J \theta(0,0))^{*} w^{*} \& w^{*} \in N(\theta(0,0) \mid \Lambda)\right] \Rightarrow w^{*}=0
$$

in terms of the generalized Jacobian $J \theta(0,0) \subset \mathbb{R}^{q(n+m)}$.

Proof. If $\theta$ is Lipschitz continuous around $(0,0)$, then (4.18) is equivalent to (4.12) by virtue of Proposition 2.8. Furthermore, condition (4.18) is always implied by (4.19) due to Corollary 2.9.

Corollary 4.6. Let $\theta$ be strictly differentiable at $(0,0)$. Then the condition

$$
\left[\left(\nabla_{y} \theta(0,0) A-\nabla_{z} \theta(0,0)\right)^{*} w^{*}=0 \& w^{*} \in N(\theta(0,0) \mid \Lambda)\right] \Rightarrow w^{*}=0
$$

is sufficient for the solution map (4.2) to (4.1), (4.11) being pseudo-Lipschitzian around $(0,0)$. This condition is necessary and sufficient for the pseudo-Lipschitzian property of (4.2) if $\operatorname{rank}\left(\nabla_{y} \theta(0,0)\right)=q \leq m$ and $\Lambda$ is regular at $(0,0)$. Proof. The sufficiency part follows directly from (4.12). If $\operatorname{rank}\left(\nabla_{y} \theta(0,0)\right)=$ $q$, then (4.13) is equivalent to (4.20) and (4.14) is fulfilled automatically. One can employ part (b) in Corollary 4.4.

Let us consider a special case of (4.1) when $Q$ is represented in the classical form of inequality and equality constraints:

$$
\begin{aligned}
& Q(z):=\left\{y \in \mathbb{R}^{m}: \theta_{i}(z, y) \leq 0 \text { for } i=1, \ldots, r\right. \text { and } \\
&\left.\theta_{i}(z, y)=0 \text { for } i=r+1, \ldots, q\right\}
\end{aligned}
$$

with smooth real-valued functions $\theta_{i}$. Let $\theta=\left(\theta_{i}\right)$,

$$
\begin{aligned}
\nabla_{z} \theta=\left(\frac{\partial \theta_{i}}{\partial z_{j}}\right), \quad \nabla_{y} \theta= & \left(\frac{\partial \theta_{i}}{\partial y_{k}}\right) \\
& \text { for } i=1, \ldots, q ; j=1, \ldots, n ; k=1, \ldots, m,
\end{aligned}
$$

and let $c_{i}(i=1, \ldots, q)$ be vector columns of the matrix

$$
C:=\nabla_{y} \theta(0,0) A-\nabla_{z} \theta(0,0) \text {. }
$$

The next assertion ensures the pseudo-Lipschitzian behavior of the solution map to (4.1), (4.21) under a generalized constraint qualification of the Mangasarian-Fromovitz type; cf. [27].

Corollary 4.7. Let the multifunction $Q$ be represented in form (4.21) where $\theta_{i}$ are strictly differentiable at $(0,0)$. Then the constraint qualification condition

$$
\begin{aligned}
{\left[\lambda_{1} c_{1}+\lambda_{2} c_{2}+\cdots+\lambda_{q} c_{q}=0\right] } & \Rightarrow \lambda_{i}=0 \text { for } i=1, \ldots, q \\
\text { if } \lambda_{i} \geq 0 \text { and } \lambda_{i} \theta_{i}(0,0) & =0 \text { for } i=1, \ldots, r
\end{aligned}
$$

with $C$ in (4.23) is sufficient for the solution map (4.2) to (4.1), (4.21) being pseudo-Lipschitzian around $(0,0)$. This condition is necessary and sufficient for the pseudo-Lipschitzness of (4.2) when the vectors $\nabla_{y} \theta_{1}(0,0), \ldots, \nabla_{y} \theta_{q}(0,0)$ are linearly independent.

Proof. It is obvious that (4.21) is a special case of (4.11) with $\theta=\left(\theta_{1}, \ldots, \theta_{q}\right)$ and with the regular (convex) set

$$
\begin{aligned}
\Lambda:=\left\{\left(w_{1}, \ldots, w_{q}\right) \in \mathbb{R}^{q}: w_{i} \leq 0\right. & \text { for } i=1, \ldots, r \text { and } \\
w_{i} & =0 \text { for } i=r+1, \ldots, q\} .
\end{aligned}
$$


One can easily compute the normal cone to (4.24) in the form

$$
\begin{aligned}
N(\theta(0,0) \mid \Lambda)=\left\{w^{*}=\left(\lambda_{1}, \ldots, \lambda_{q}\right) \in \mathbb{R}^{q}: \lambda_{i} \geq 0\right. \\
\\
\text { and } \left.\lambda_{i} \theta_{i}(0,0)=0 \text { for } i=1, \ldots, r\right\} .
\end{aligned}
$$

Therefore, the results being proved for case (4.21) follow directly from Corollary 4.6.

Now we consider the setting when the multifunction $Q$ in (4.1) is a subdifferential mapping for some extended-real-values function $\varphi: \mathbb{R}^{n} \rightarrow \overline{\mathbb{R}}$, i.e.,

$$
Q(z):= \begin{cases}\partial^{-} \varphi(z) & \text { if }|\varphi(z)|<\infty, \\ \varnothing & \text { otherwise }\end{cases}
$$

in terms of the subdifferential (2.13). This includes, in particular, the cases of variational inequalities and complementarity problems when $\varphi(z)=\delta(z, \Omega)$ is the indicator function of some convex set $\Omega$; see $\S 1$. Theorem 4.1 implies the following criterion of the Lipschitzian stability for such generalized equations stated in terms of the second order subdifferential (2.14).

Corollary 4.8. Let the multifunction $Q$ be represented in the subdifferential form (4.25). Then the condition

$$
\left[0 \in A^{*} y^{*}+\partial^{2,-} \varphi(0,0)\left(y^{*}\right)\right] \Rightarrow y^{*}=0
$$

is necessary and sufficient for the solution map (4.2) to (4.1), (4.25) being pseudo-Lipschitzian around $(0,0)$.

Proof. Note that the set-valued mapping (4.25) has closed graph for any extended-real-valued function $\varphi$, due to the robustness property of the subdifferential (2.13); see Remark 2.4. For such multifunctions $Q$, conditions (4.4) and (4.26) are equivalent by virtue of Definition (2.14). Therefore, the assertion being proved follows from Theorem 4.1 .

Remark 4.9. If one replaces the coderivative (2.5) in (4.4) by the Clarke coderivative (2.6), then the condition obtained

$$
\left[0 \in A^{*} y^{*}+D_{C}^{*} Q(0,0)\left(y^{*}\right)\right] \Rightarrow y^{*}=0
$$

is reduced to Rockafellar's sufficient condition for (4.2) being pseudoLipschitzian around $(0,0)$; cf. [34, Corollary 3.12]. In particular, if $Q$ is the subdifferential mapping for a convex function $\varphi$, then the Clarke coderivative in (4.27) is reduced to (2.17) and coincides with the second order derivative of $\varphi$ employed by Aubin [1] in his applications to sensitivity analysis of convex minimization problems.

It turns out that the sufficient condition (4.27) is much more restrictive than criterion (4.4) and does not hold for a broad class of generalized equations important for applications. In particular, if $m=n$ and the graph of $Q$ happens to be a Lipschitzian manifold of dimension $n$ around $(0,0)$ (this is always the case when (4.1) is a variational inequality), then the Clarke normal cone $N_{C}((0,0) \mid \operatorname{gph} Q)$ is always a linear subspace $S$ having dimension at least $n$; see Proposition 3.1 above summarizing results in Rockafellar [35]. But in this setting the sufficient condition (4.27) easily implies that dimension of $S$ is exactly $n$ and the graph of $Q$ must be strictly smooth at $(0,0)$; see again Proposition 3.1 and also [34, Remark 3.13]. Moreover, if $Q=\partial \varphi$ with a closed 
proper convex function on $\mathbb{R}^{n}$, then the latter property corresponds to some second order differentiability of $\varphi$ which is very close to the classical contents; see $[35, \S 4]$ for more details. Therefore, condition (4.27) stated in terms of the Clarke normal case cannot actually cover the case of variational inequalities and complementarity problems where $\varphi$ is the indicator function of some convex set. In contrast to this, criterion (4.26) does not require any special properties of $\varphi$ and allows us to provide an effective sensitivity analysis of the problems under consideration. Now consider a typical example of employing criterion (4.26) in the framework of perturbed variational inequalities.

Example 4.10. Considering the following perturbed variational inequality

$$
\text { find } z \geq 0 \text { s.t. }(a z+p)(\omega-z) \geq 0 \text { for all } \omega \geq 0 \text {, }
$$

we can see that (4.28) is equivalent to the linear generalized equation (4.1) with $z \in \mathbb{R}, A=a$, and

$$
Q(z)=\partial \varphi(z)= \begin{cases}0 & \text { if } z>0 \\ \mathbb{R}_{-} & \text {if } z=0 \\ \varnothing & \text { if } z<0\end{cases}
$$

for the indicator convex function $\varphi(z):=\delta(z, \Omega)$ with $\Omega:=\mathbb{R}_{+}$. Let us compute

$$
D^{*} Q(0,0)(u)=\{v \in \mathbb{R}:(v,-u) \in N((0,0) \mid \operatorname{gph} Q)\}
$$

for the multifunction $Q$ in (4.29) with nonconvex graph

$$
\operatorname{gph} Q=\left\{(z, y) \in \mathbb{R}^{2}: z \geq 0, y \leq 0 \& z y=0\right\} .
$$

One easily has that $N((0,0) \mid \operatorname{gph} Q)=\left\{(v, u) \in \mathbb{R}^{2}: v \leq 0, u \geq 0\right\}$ and

$$
D^{*} Q(0,0)(u)= \begin{cases}0 & \text { if } u>0, \\ \mathbb{R} & \text { if } u=0, \\ \mathbb{R}_{-} & \text {if } u<0\end{cases}
$$

Putting the result to the adjoint generalized equation (4.3) for (4.28), we can conclude that this equation has only the trivial solution if and only if $a>0$. According to Theorem 4.1 (or Corollary 4.8), the condition obtained is necessary and sufficient for the pseudo-Lipschitzness of the solution map to perturbed variational inequality $(4.28)$ at $(0,0)$. Note that

$$
N((0,0) \mid \operatorname{gph} Q)=\mathbb{R}^{2} \text { and } D^{*} Q(0,0)(u) \equiv \mathbb{R}
$$

for $Q$ in (4.29), i.e., condition (4.27) carries no information about the Lipschitzian stability of (4.28).

Now let us consider the following class of linear generalized equations with nonlinear additive parameters:

$$
0 \in A z+h(p)+Q(z)
$$

where $A$ and $Q$ are the same as in (4.1) and $h: \mathbb{R}^{k} \rightarrow \mathbb{R}^{m}$ is a continuous vector function of the parameter $p$ such that $h(0)=0$. The corresponding set of solutions to (4.30) is

$$
\Sigma(p):=\left\{z \in \mathbb{R}^{n}: 0 \in A z+h(p)+Q(z)\right\}
$$


We study the pseudo-Lipschitzian behavior of the solution map (4.31) around the points $(p, 0)$ with

$$
p \in \bar{P}:=h^{-1}(0)=\left\{p \in \mathbb{R}^{k}: h(p)=0\right\} .
$$

Note that such points satisfy (4.30).

Theorem 4.11. (i) If the function $h$ is locally Lipschitzian around some $p \in$ $\bar{P}$, then condition (4.4) is sufficient for (4.31) being pseudo-Lipschitzian around $(p, 0)$. Conversely if the inverse multifunction $h^{-1}$ is locally bounded around 0 and

$$
\operatorname{Ker} D^{*} h(p)=\{0\} \quad \forall p \in \bar{P},
$$

then condition (4.4) is necessary for (4.31) being pseudo-Lipschitzian around any $(p, 0)$ with $p \in \bar{P}$.

(ii) If $h$ is Lipschitz continuous around $p=0$ and its inverse admits a singlevalued Lipschitz continuous selection $\gamma(v) \in h^{-1}(v)$ with $\gamma(0)=0$ in some neighborhood of $v=0$ (in particular, $h$ is a locally Lipschitzian homeomorphism around 0), then condition (4.4) is necessary and sufficient for (4.31) being pseudo-Lipschitzian around $(0,0)$.

Proof. Let us prove (i). Along with (4.30), we consider the original generalized equation (4.1) with the linear parameter $v=p$. One can observe the interrelation

$$
\Sigma(p)=\Gamma(h(p))
$$

between the solution map (4.31) to (4.30) and the solution map (4.2) to (4.1) with the parameter $v$. If the function $h$ is locally Lipschitz around some point $p \in \bar{P}$ and $\Gamma$ is pseudo-Lipschitzian around $(0,0)$, then the multifunction $\Sigma$ will be pseudo-Lipschitzian around $(p, 0)$ according to Proposition 3.11 with a single-valued and Lipschitz continuous internal function. Therefore, criterion (4.4), ensuring the pseudo-Lipschitzness of the multifunction (4.2) around $(0,0)$ by virtue of Theorem 4.1 , provides a sufficient condition for the pseudo-Lipschitzness of the solution map (4.31).

Let us prove the necessity part in assertion (i) of the theorem. If condition (4.32) is fulfilled and the inverse mapping $h^{-1}$ is locally bounded around 0 , then this multifunction will be nonempty-compact-valued and Lipschitzian in some neighborhood of 0 by virtue of Corollary 3.8(iii). In this setting we have the representation

$$
\Gamma(v)=\Sigma\left(h^{-1}(v)\right)
$$

around $v=0$ and can employ Proposition 3.11 for the case of locally Lipschitzian internal multifunction. According to this proposition, the pseudoLipschitzness of $\Sigma$ around any $(p, 0)$ with $p \in \bar{P}$ and the locally Lipschitzness of $h^{-1}$ around 0 imply the pseudo-Lipschitzness of $\Gamma$ in $(4.2)$ around $(0,0)$. Due to Theorem 4.1, the latter means that condition (4.4) must be fulfilled.

Now we prove (ii). The sufficiency part in (ii) follows from (i) as $p=0$. For proving the necessity in (ii), let us consider a neighborhood $U$ of $p=0$ where the solution map (4.31) satisfies the pseudo-Lipschitzian property around $(0,0)$. Taking the Lipschitzian selection $\gamma$ of $h^{-1}$, one can find neighborhood $V$ of $v=0$ such that $\gamma(V) \subset U$ and

$$
\Gamma(v)=\Sigma(\gamma(v)) \text { for } v \in V .
$$


Employing Proposition 3.11, again we conclude that $\Gamma$ in (4.2) is a pseudoLipschitzian multifunction around $(0,0)$. Therefore, condition $(4.4)$ is fulfilled. This ends the proof of the theorem.

Remark 4.12. One can see that the two assertions in Theorem 4.11 are independent even in the case when $\bar{P}=\{0\}$. Indeed, in this case the condition (4.32) is equivalent to the multifunction $h^{-1}$ being Lipschitz continuous around 0 in the Hausdorff metric. But this does not imply the existence of a locally Lipschitzian selection (see, e.g., [2, §9.4]). Conversely, simple examples show that the existence of a Lipschitzian selection does not ensure the Lipschitzian or pseudo-Lipschitzian properties of multifunctions. Both assertions in Theorem 4.11 are equivalent if $h$ is a locally Lipschitzian homeomorphism around 0 . Note that a necessary and sufficient condition for the latter property has been recently found by Kummer [14] in terms of Thibault's generalized directional derivative of $h$ [38]. This condition implies (4.32) as $p=0$.

Due to Proposition 2.8, one can reduce (4.32) to

$$
\left[0 \in \partial^{-}\left\langle y^{*}, h\right\rangle(p)\right] \Rightarrow y^{*} \quad \forall p \in \bar{P}
$$

when $h$ is Lipschitz continuous around $p \in \bar{P}$. The latter condition holds automatically if every matrix belonging to the generalized Jacobian $J h(p)$ has maximal rank, i.e.,

$$
\operatorname{rank}(J h(p))=m \leq k \quad \forall p \in \bar{P}
$$

For $m=k$ in (4.33), we obtain the following concretization of Theorem 4.11.

Corollary 4.13. Let $h: \mathbb{R}^{k} \rightarrow \mathbb{R}^{k}$ be Lipschitz continuous around $p=0$ and let every matrix belonging to the generalized Jacobian $J h(0)$ be nonsingular. Then condition (4.4) is necessary and sufficient for the solution map (4.31) to be pseudo-Lipschitzian around $(0,0)$.

Proof. If (4.33) is fulfilled for $p=0$ with $m=k$, then, according to the inverse function theorem of Clarke [4, Theorem 7.1.1], the Lipschitz continuous function $h$ admits a single-valued and locally Lipschitzian inverse around 0 . We meet all the assumptions in both assertions of Theorem 4.11.

According to Corollaries 4.2-4.8, the results obtained imply various sufficient conditions as well as necessary and sufficient conditions for the pseudoLipschitzian property of the solution map (4.31) in the cases of special representations of the multifunction $Q$.

\section{NONLINEAR SMOOTH GENERALIZED EQUATIONS}

In this section we study the nonlinear perturbed generalized equations

$$
0 \in f(p, z)+Q(z)
$$

depending on a parameter $p \in \mathbb{R}^{k}$ where $f: \mathbb{R}^{k} \times \mathbb{R}^{n} \rightarrow \mathbb{R}^{m}$ is smooth in the decision variable $z$. In what follows we assume that $Q: \mathbb{R}^{n} \Rightarrow \mathbb{R}^{m}$ is an arbitrary multifunction of closed graph, the function $f$ is continuous around the given point $(\bar{p}, \bar{z})$ and $-f(\bar{p}, \bar{z}) \in Q(\bar{z})$. Let us consider the solution map

$$
\Sigma(p):=\left\{z \in \mathbb{R}^{n}: 0 \in f(p, z)+Q(z)\right\}
$$


and study the pseudo-Lipschitzian property of (5.2) around the point $(\bar{p}, \bar{z})$ satisfying (5.1). Following Robinson [28, 29], we define the linear generalized equation

$$
0 \in f(\bar{p}, \bar{z})+\nabla_{z} f(\bar{p}, \bar{z})(z-\bar{z})-y+Q(z)
$$

depending on a linear parameter $y \in \mathbb{R}^{m}$ and call (5.3) the linearization of (5.1) at the point $(\bar{p}, \bar{z})$. One can see that the set of solutions

$$
\Gamma(y):=\left\{z \in \mathbb{R}^{n}: 0 \in f(\bar{p}, \bar{z})+\nabla_{z} f(\bar{p}, \bar{z})(z-\bar{z})-y+Q(z)\right\}
$$

to the linearized equation (5.3) coincides with the value $L^{-1}(y)$ of the inverse multifunction to the operator

$$
L(z):=f(\bar{p}, \bar{z})+\nabla_{z} f(\bar{p}, \bar{z})(z-\bar{z})+Q(z) .
$$

Some relationships between Lipschitzian properties of the solution maps (5.2) and (5.4) have been studied in [6, 28, 29]. In [28], Robinson obtained conditions under which the so-called upper Lipschitzian property of (5.4) implies the same property of the solution map (5.2). Another paper of Robinson [29] contains conditions ensuring the single-valuedness and locally Lipschitzness of (5.2) provided that the solution map to the linearized equation enjoys these properties. In the recent paper [6], Dontchev and Hager have proved, employing a contractive mapping principle for multifunctions, that the pseudo-Lipschitzian property of the solution map (5.4) induces such a property for (5.2) in general settings. This allows us to obtain effective sufficient conditions for (5.2) to be pseudo-Lipschitzian using the pseudo-Lipschitzian criteria for linear generalized equations in $\S 4$.

In the following theorem we assume that $f$ is strictly differentiable in $z$ at $(\bar{p}, \bar{z})$, i.e.,

$$
\lim _{\substack{z, z^{\prime} \overrightarrow{\bar{p}} \overline{\bar{p}} \\ p \rightarrow \bar{p}}}\left[\frac{f(p, z)-f\left(p, z^{\prime}\right)-\nabla_{z} f(\bar{p}, \bar{z})\left(z-z^{\prime}\right)}{\left\|z-z^{\prime}\right\|}\right]=0 .
$$

It happens, in particular, when $f$ is differentiable in $z$ around $(\bar{p}, \bar{z})$ and $\nabla_{z} f$ is continuous in both $p$ and $z$ at this point. Recall also that $f$ is said to be locally Lipschitzian in $p$ uniformly in $z$ around $(\bar{p}, \bar{z})$ if there exist a number $l \geq 0$ and neighborhoods $U$ of $\bar{p}$ and $V$ of $\bar{z}$ such that

$$
\left\|f(p, z)-f\left(p^{\prime}, z\right)\right\| \leq l\left\|p-p^{\prime}\right\|
$$

for any $p, p^{\prime} \in U$, and $z \in V$.

Theorem 5.1. Let $f$ be strictly differentiable in $z$ at $(\bar{p}, \bar{z})$ and let it be locally Lipschitzian in $p$ uniformly in $z$ around this point. Then the solution map (4.2) is pseudo-Lipschitzian around $(\bar{p}, \bar{z})$ if the adjoint generalized equation

$$
0 \in\left(\nabla_{z} f(\bar{p}, \bar{z})\right)^{*} y^{*}+D^{*} Q(\bar{z},-f(\bar{p}, \bar{z}))\left(y^{*}\right)
$$

has only the trivial solution $y^{*}=0$.

Proof. It follows from [6, Theorem 4.1] that, under the assumptions imposed on $f$, the pseudo-Lipschitzness of multifunction $(5.4)$ around $(0, \bar{z})$ implies the pseudo-Lipschitzness of the solution map (5.2) around $(\bar{p}, \bar{z})$. Employing 
Theorem 4.1 and using an elementary representation of the coderivative (2.5) for the multifunction

$$
\Phi(z):=f(\bar{p}, \bar{z})+Q(x+\bar{z})
$$

at $(0,0)$, one can easily conclude that the condition

$$
\left[0 \in\left(\nabla_{z} f(\bar{p}, \bar{z})\right)^{*} y^{*}+D^{*} Q(\bar{z},-f(\bar{p}, \bar{z}))\left(y^{*}\right)\right] \Rightarrow y^{*}=0
$$

is necessary and sufficient for (5.4) being pseudo-Lipschitzian around $(0, \bar{z})$. Therefore, (5.7) provides a sufficient condition for the pseudo-Lipschitzness of the solution map (5.2) around $(\bar{p}, \bar{z})$.

Now we obtain some corollaries of Theorem 4.1 based on Corollaries 4.2-4.8 of Theorem 4.1 for the linearized equation (5.3).

Corollary 5.2. Let $f$ satisfy the assumptions in Theorem 5.1 and let $Q$ in (5.1) have convex graph. If condition (4.7) is fulfilled for the operator $L$ in (5.5), then the solution map (5.2) is pseudo-Lipschitzian around $(\bar{p}, \bar{z})$.

Proof. This follows from Theorem 5.1 by virtue of Corollary 4.2 for the linearized operator $L$ in (5.5).

Corollary 5.3. Let $f$ satisfy the assumptions in Theorem 5.1 and let $Q$ be represented in form (4.9) with some closed and convex sets $E$ and $\Omega$. Then the condition

$$
0 \in \operatorname{int}\left\{f(\bar{p}, \bar{z})+\nabla_{z} f(\bar{p}, \bar{z})(\Omega-\bar{z})+E\right\}
$$

is sufficient for the solution map (5.2) to (5.1), (4.9) to be pseudo-Lipschitzian around $(\bar{p}, \bar{z})$.

Proof. This follows from Theorem 5.1 due to Corollary 5.3 for the linear generalized equation (5.3).

Note that (5.8) is the Robinson regularity condition first introduced and utilized in [27] for the case of $E$ being a convex cone.

Corollary 5.4. Let $f$ satisfy the assumptions in Theorem 5.1 and let $Q$ admit representation (4.11) where $\Lambda \in \mathbb{R}^{q}$ is a closed set and $\theta: \mathbb{R}^{n} \times \mathbb{R}^{m} \rightarrow \mathbb{R}^{q}$ is a vector function continuous around the point $(\bar{z},-f(\bar{p}, \bar{z}))$ with $\theta(\bar{z},-f(\bar{p}, \bar{z}))$ $\in \Lambda$. Then:

(i) The condition

$$
\begin{aligned}
& {\left[\left(\left(\nabla_{z} f(\bar{p}, \bar{z})\right)^{*} y^{*}, y^{*}\right) \in D^{*} \theta(\bar{z},-f(\bar{p}, \bar{z}))\left(w^{*}\right) \&\right.} \\
& \left.\quad w^{*} \in N(\theta(\bar{x},-f(\bar{p}, \bar{z})) \mid \Lambda)\right] \Rightarrow w^{*}=0 \& y^{*}=0
\end{aligned}
$$

is sufficient of the solution map (5.2) to (5.1), (4.11) to be pseudoLipschitzian around $(\bar{p}, \bar{z})$.

(ii) If $\theta$ is Lipschitz continuous around $(\bar{z},-f(\bar{p}, \bar{z}))$, then the sufficient condition (5.9) is equivalent to

$$
\begin{array}{r}
{\left[\left(\left(\nabla_{z} f(\bar{p}, \bar{z})\right)^{*} y^{*}, y^{*}\right) \in \partial^{-}\left\langle w^{*}, \theta\right\rangle(\bar{z},-f(\bar{p}, \bar{z})) \&\right.} \\
\left.w^{*} \in N(\theta(\bar{z},-f(\bar{p}, \bar{z})) \mid \Lambda)\right] \Rightarrow w^{*}=0
\end{array}
$$

which is automatically fulfilled when

$$
\begin{array}{r}
{\left[\left(\left(\nabla_{z} f(\bar{p}, \bar{z})\right)^{*} y^{*}, y^{*}\right) \in(J \theta(\bar{z},-f(\bar{p}, \bar{z})))^{*} w^{*} \&\right.} \\
\left.w^{*} \in N(\theta(\bar{z},-f(\bar{p}, \bar{z})) \mid \Lambda)\right] \Rightarrow w^{*}=0
\end{array}
$$

in terms of the generalized Jacobian of $\theta$ at $(\bar{z},-f(\bar{p}, \bar{z}))$. 
(iii) If $\theta$ is strictly differentiable at $(\bar{z},-f(\bar{p}, \bar{z}))$, then the sufficient condition (5.10) is equivalent to

$$
\begin{gathered}
{\left[\left(\nabla_{y} \theta(\bar{z},-f(\bar{p}, \bar{z})) \nabla_{z} f(\bar{p}, \bar{z})-\nabla_{z} \theta(\bar{z},-f(\bar{p}, \bar{z}))\right)^{*} w^{*}=0 \&\right.} \\
\left.w^{*} \in N(\theta(\bar{z},-f(\bar{p}, \bar{z})) \mid \Lambda)\right] \Rightarrow w^{*}=0
\end{gathered}
$$

which is automatically fulfilled when

$$
\operatorname{rank}\left(\nabla_{y} \theta(\bar{z},-f(\bar{p}, \bar{z})) \nabla_{z} f(\bar{p}, \bar{z})-\nabla_{z} \theta(\bar{z},-f(\bar{p}, \bar{z}))\right)=q .
$$

Proof. Assertion (i) follows from Theorem 5.1 and the sufficiency part in Corollary 4.4 for (5.3). Assertions (ii) and (iii) follow from Propositions 2.6 and 2.5(ii) respectively.

Let us obtain a specification of assertion (iii) in Corollary 5.4 for the classical representation (4.21) of the multifunction $Q$ in (5.1). Using notation (4.22), we consider the matrix

$$
C:=\nabla_{y} \theta(\bar{z},-f(\bar{p}, \bar{z})) \nabla_{z} f(\bar{p}, \bar{z})-\nabla_{z} \theta(\bar{z},-f(\bar{p}, \bar{z}))
$$

and denote by $c_{i}(i=1, \ldots, q)$ its vector columns.

Corollary 5.5. Let $f$ satisfy the assumptions in Theorem 5.1 and let $Q$ admit representation (4.21) with real-valued functions $\theta_{i}$ strictly differentiable at $(\bar{z},-f(\bar{p}, \bar{z}))$. Then the condition

$$
\begin{gathered}
{\left[\lambda_{1} c_{1}+\lambda_{2} c_{2}+\cdots+\lambda_{q} c_{q}=0\right] \Rightarrow \lambda_{i}=0 \text { for } i=1, \ldots, q} \\
\text { if } \lambda_{i} \geq 0 \text { and } \lambda_{i} \theta_{i}(\bar{z},-f(\bar{p}, \bar{z}))=0 \text { for } i=1, \ldots, r
\end{gathered}
$$

with the matrix $C$ in (5.13) is sufficient for the solution map (5.2) to (5.1), (4.21) to be pseudo-Lipschitzian around $(\bar{p}, \bar{z})$.

Proof. This follows from assertion (iii) in Corollary 5.4 for $\Lambda$ as in (4.24).

Corollary 5.6. Let $f$ satisfy the assumptions in Theorem 5.1 and let $Q$ be the subdifferential mapping (4.25) with an extended-real-valued function $\varphi$. Then the condition

$$
\left[0 \in\left(\nabla_{z} f(\bar{p}, \bar{z})\right)^{*} y^{*}+\partial^{2,-} \varphi(\bar{z},-f(\bar{p}, \bar{z}))\left(y^{*}\right)\right] \Rightarrow y^{*}=0
$$

is sufficient for the solution map (5.2) to (5.1), (4.25) to be pseudo-Lipschitzian around $(\bar{p}, \bar{z})$.

Proof. This follows from Theorem 5.1 and Corollary 4.8 for (5.3).

Remark 5.7. The sufficient condition in Theorem 5.1 for the solution map (5.2) to be pseudo-Lipschitzian around $(\bar{p}, \bar{z})$ has been obtained in Mordukhovich [23, Theorem 5.8] under the assumption that $f$ is strictly differentiable at $(\bar{p}, \bar{z})$ with respect to both variables $(p, z)$. This was proved by reducing the generalized equation (5.1) to a general constraint system in [23] without reference to the linearized equation (5.3). Using the procedure in this section, we remove the smoothness assumption for $f$ with respect to $p$ in the sufficient condition (5.7). On the other hand, the approach in [23] allows us to single out general settings when (5.7) is a necessary and sufficient condition for the pseudoLipschitzness of (5.2) while $f$ is smooth in $(p, z)$. If $f$ may be nonsmooth in $p$ but it is separated with respect to $(p, z)$, i.e.,

$$
f(p, z)=b(z)+h(p),
$$


then another setting when (5.7) is a necessary and sufficient condition for the pseudo-Lipschitzness of (5.2) can be obtained by using the procedure in the proof of Theorem 4.11. The next theorem summarizes all the results in this direction.

Theorem 5.8. (i) Condition (5.7) is necessary and sufficient for the solution map (5.2) to the generalized equation (5.1) to be pseudo-Lipschitzian around $(\bar{p}, \bar{z})$ if one of the following groups of assumptions $(\mathrm{H} 1)-(\mathrm{H} 3)$ holds:

(H1) $f$ is strictly differentiable in $(p, z)$ at the point $(\bar{p}, \bar{z})$ and the matrix $\nabla_{p} f(\bar{p}, \bar{z})$ is quadratic and nonsingular, i.e., $\operatorname{rank}\left(\nabla_{p} f(\bar{p}, \bar{z})\right)=m=$ $k$;

(H2) $f$ is strictly differentiable in $(p, z)$ at $(\bar{p}, \bar{z}), \operatorname{rank}\left(\nabla_{p} f(\bar{p}, \bar{z})\right)=m<$ $k$, and $Q$ is differentially regular at $(\bar{z},-f(\bar{p}, \bar{z}))$;

(H3) $f$ is represented in the form (5.16) where $b$ is strictly differentiable at $\bar{z}$ while $h$ is Lipschitz continuous around $\bar{p}$ and its inverse $h^{-1}$ admits a single-valued Lipschitz continuous selection around $h(\bar{p})$ passing through $(h(\bar{p}), \bar{p})$ (in particular, $h$ is a locally Lipschitzian homeomorphism around $\bar{p})$.

(ii) Suppose that the following hypothesis is fulfilled:

(H4) $f$ admits representation (5.16) where $b$ is strictly differentiable at $\bar{z}$, $h$ is Lipschitz continuous around any point $p$ with $h(p)=h(\bar{p})$, the inverse multifunction $h^{-1}$ is locally bounded around $h(\bar{p})$ and assumption (4.32) holds with

$$
\bar{P}:=h^{-1}(h(\bar{p}))=\left\{p \in \mathbb{R}^{k}: h(p)=h(\bar{p})\right\} .
$$

Then condition (5.7) is necessary and sufficient for the solution map (5.2) to be pseudo-Lipschitzian around any point $(p, \bar{z})$ with $p \in \bar{P}$.

Proof. Assertion (i) under hypotheses (H1) and (H2) follows directly from [23, Theorem 5.8]. The sufficiency parts in (i) and (ii) in the case of representation (5.16) are implied by Theorem 5.1. For proving the necessity of (5.7) for the pseudo-Lipschitzness of (5.2) under hypotheses (H3) and (H4), let us consider the generalized equation

$$
0 \in b(z)+v+Q(z)
$$

with the linear additive parameter $v \in \mathbb{R}^{m}$. One can observe that the function $f(z, v):=b(z)+v$ satisfies $(\mathrm{H} 1)$ at the point $(h(\bar{p}), \bar{z})$. Therefore, condition (5.7) is necessary and sufficient for the pseudo-Lipschitzian property of the solution map to $(5.17)$ at $(h(\bar{p}), \bar{z})$. Now using the same arguments as in the proof of Theorem 4.11 , we conclude that (5.7) is a necessary condition for the pseudo-Lipschitzian property of (5.2) around the corresponding points under assumptions $(\mathrm{H} 3)$ and $(\mathrm{H} 4)$ respectively.

The results obtained allow us to establish the equivalence between the pseudoLipschitzness of the solution maps to the nonlinear generalized equation (5.1) and its linearization (5.3) under the assumptions in Theorem 5.8.

Corollary 5.9. (i) Let one of the assumption groups $(\mathrm{H} 1)-(\mathrm{H} 3)$ be fulfilled. Then the solution map (5.2) to the nonlinear generalized equation (5.1) is pseudoLipschitzian around $(\bar{p}, \bar{z})$ if and only if the solution map (5.4) to the linearized equation (5.3) is pseudo-Lipschitzian around $(0, \bar{z})$. 
(ii) Let (H4) be fulfilled. Then for the pseudo-Lipschitzian property of the solution map (5.2) to (5.2), (5.16) around any point $(p, \bar{z})$ with $h(p)=h(\bar{p})$, it is necessary and sufficient that the solution map to the linearized equation (5.3), (5.16) be pseudo-Lipschitzian around $(0, \bar{z})$.

Proof. This follows from Theorems 5.8, 4.1, and 4.11 which provide the same criteria for the pseudo-Lipschitzness of the solution maps to the initial and linearized generalized equations under the assumptions made.

Let us single out a special case of the results obtained when both (H3) and (H4) are fulfilled with $\bar{P}=\{\bar{p}\}$ in (H4).

Corollary 5.10. Let $f$ admit representation (5.16) where $b$ is continuous differentiable around $\bar{z}$ and $h$ is Lipschitz continuous around $\bar{p}$ with

$$
\operatorname{rank}(J h(\bar{p}))=m=k
$$

in terms of generalized Jacobian of $h$ at $\bar{p}$. Then condition (5.7) is necessary and sufficient for the solution map (5.2) to be pseudo-Lipschitzian around $(\bar{p}, \bar{z})$ which is equivalent to the pseudo-Lipschitzian property of the solution map (5.4) around $(0, \bar{z})$.

Proof. According to Clarke [4, Theorem 7.1], condition (5.18) implies that $h$ is a Lipschitzian homeomorphism around $h(\bar{p})$, i.e., the fulfillment of (H3). On the other hand, (5.18) implies (4.32) with $\bar{P}=\{\bar{p}\}$. Therefore, the results being proved follow from both assertions in Theorem 5.8 and Corollary 5.9.

Note that Clarke's condition (5.18) can be replaced in Corollary 4.10 by a more general Kummer condition [14] providing a criterion for $h$ to be a locally Lipschitzian homeomorphism around $\bar{p}$. Both Clarke's and Kummer's conditions imply the main assumption (4.32) in (H4) with $\bar{P}=\{\bar{p}\}$. Note also that hypotheses (H3) and (H4) are independent even in the case of $\bar{P}=\{\bar{p}\}$; see Remark 4.12.

Now we obtain some effective necessary and sufficient conditions for the pseudo-Lipschitzian property of the solution map to the nonlinear generalized equation (5.1) with the multifunction $Q$ of special structure. For convenience of the statements, we simplify (H4) assuming that $\bar{P}=\{\bar{p}\}$. In this case the conclusions in both assertions (i) and (ii) of Theorem 5.8 and Corollary 5.9 coincide.

Corollary 5.11. Let $f$ in (5.1) satisfy one of the hypotheses $(\mathrm{H} 1)-(\mathrm{H} 3)$ or $(\mathrm{H} 4)$ with $\bar{P}=\{\bar{p}\}$ and let $Q$ have convex graph. Then condition (4.7) with the operator $L$ in (5.5) is necessary and sufficient for the solution map (5.2) to be pseudo-Lipschitzian around $(\bar{p}, \bar{z})$.

Proof. This follows from Corollaries 5.9 and 4.2 for the linearized generalized equation (5.3).

Corollary 5.12. Let $f$ in (5.1) satisfy one of the hypotheses $(\mathrm{H} 1)-(\mathrm{H} 3)$ or $(\mathrm{H} 4)$ with $\bar{P}=\{\bar{p}\}$ and let $Q$ admit representation (4.9) with some closed and convex sets $E$ and $\Omega$. Then condition (5.8) is necessary and sufficient for the solution map (5.2) to be pseudo-Lipschitzian around $(\bar{p}, \bar{z})$.

Proof. This follows from Corollaries 5.9 and 4.3 for the linearized generalized equation (5.3). 
Corollary 5.13. Let $f$ in (5.1) satisfy one of the hypotheses $(\mathrm{H} 1)-(\mathrm{H} 3)$ or $(\mathrm{H} 4)$ with $\bar{P}=\{\bar{p}\}$ and let $Q$ admit representation (4.11) where $\theta: \mathbb{R}^{n} \times \mathbb{R}^{m} \rightarrow \mathbb{R}^{q}$ is strictly differentiable at $(\bar{z},-f(\bar{p}, \bar{z}))$. Then the condition

$$
\begin{array}{r}
{\left[\left(\nabla_{y} \theta(\bar{z},-f(\bar{p}, \bar{z})) \nabla_{z} f(\bar{p}, \bar{z})-\nabla_{z} \theta(\bar{z},-f(\bar{p}, \bar{z}))\right)^{*} w^{*}=0 \&\right.} \\
\left.w^{*} \in N(\theta(\bar{z},-f(\bar{p}, \bar{z})) \mid \Lambda)\right] \Rightarrow\left(\nabla_{y} \theta(\bar{z},-f(\bar{p}, \bar{z}))^{*} w^{*}=0\right.
\end{array}
$$

is necessary and sufficient for the solution map (5.2) to be pseudo-Lipschitzian around $(\bar{p}, \bar{z})$ when either

(a) $\nabla \theta(\bar{z},-f(\bar{p}, \bar{z}))$ is quadratic $(q=n+m)$ and nonsingular, or

(b) $\Lambda$ is regular at $\theta(\bar{z},-f(\bar{p}, \bar{z}))$ and

$$
\operatorname{Ker}(\nabla \theta(\bar{z},-f(\bar{p}, \bar{z})))^{*} \cap N(\theta(\bar{z},-f(\bar{p}, \bar{z})) \mid \Lambda)=\{0\} .
$$

Proof. This follows from Remark 4.9 and Corollary 4.4 for the linearized equation $(5.3)$ when $\theta$ is strictly differentiable at $(\bar{z},-f(\bar{p}, \bar{z}))$.

Corollary 5.14. Let $f$ and $Q$ satisfy the general assumptions of Corollary 5.12 with $\Lambda$ regular at $\theta(\bar{z},-f(\bar{p}, \bar{z}))$. If

$$
\operatorname{rank}\left(\nabla_{y} \theta(\bar{z},-f(\bar{p}, \bar{z}))\right)=q \leq m,
$$

then condition (5.12) is necessary and sufficient for the pseudo-Lipschitzian property of the solution map (5.2) around $(\bar{p}, \bar{z})$.

Proof. If (5.21) is fulfilled, then (5.19) is equivalent to (5.12) while (5.20) holds automatically. Therefore, the result follows from Corollary 5.12 (cf. also Corollary 4.6).

Let us formulate a necessary and sufficient condition for the pseudo-Lipschitz-

ness of the solution map to (5.1) when $Q$ admits the classical representation (4.21). Consider the matrix $C$ in $(5.13)$ and denote by $c_{i}(i=1, \ldots, q)$ its vector columns.

Corollary 5.15. Let $f$ in (5.1) satisfy the assumptions in Corollary 5.14 and let $Q$ admit representation (4.21) with real-valued functions $\theta_{i}$ strictly differentiable at $(\bar{z},-f(\bar{p}, \bar{z}))$. If vectors

$$
\nabla_{y} \theta_{1}(\bar{z},-f(\bar{p}, \bar{z})), \nabla_{y} \theta_{2}(\bar{z},-f(\bar{p}, \bar{z})), \ldots, \nabla_{y} \theta_{q}(\bar{z},-f(\bar{p}, \bar{z}))
$$

are linearly independent, then condition (5.14) with the matrix $C$ in (5.13) is necessary and sufficient for (5.2) to be pseudo-Lipschitzian around $(\bar{p}, \bar{z})$.

Proof. This follows from Corollary 5.14 when $\Lambda$ is defined by (4.24); cf. also Corollary 4.7.

The next assertion contains a criterion of the Lipschitzian stability for the generalized equation (5.1) in the case when $Q$ is the subdifferential mapping (4.25). This includes the cases of nonlinear variational inequalities and complementarity problems; see $\S 7$ for further considerations.

Corollary 5.16. Let $f$ in (5.1) satisfy one of the hypotheses $(\mathrm{H} 1)-(\mathrm{H} 3)$ or $(\mathrm{H} 4)$ with $\bar{P}=\{\bar{p}\}$ and let $Q$ be a subdifferential mapping of the form (4.25) with an extended-real-valued function $\varphi$. Then condition (5.15) is necessary and sufficient for the solution map (5.2) to be pseudo-Lipschitzian around $(\bar{p}, \bar{z})$.

Proof. This follows from Corollaries 5.9 and 4.8 for the linearized generalized equation (5.3). 
Now we consider a special case of the generalized equation (5.1) when $Q(z) \equiv$ $\{0\}$. The solution map (5.2) to such a generalized equation is reduced to

$$
\Sigma(p)=\left\{z \in \mathbb{R}^{n}: f(p, z)=0\right\} .
$$

This means that $\Sigma(p)$ is actually an implicit mapping defined by the equation $f(p, z)=0$. In contrast to the classical implicit function theorem and its recent generalizations (see, e.g., Robinson [31] and references therein), we consider here a general setting when the mapping $\Sigma$ may be multivalued, i.e., (5.22) defines an implicit multifunction. The next result contains a general sufficient condition for the pseudo-Lipschitzian property of the implicit multifunction (5.22) and provides some additional assumptions when the condition obtained is necessary and sufficient for this property.

Theorem 5.17. (i) Let $f$ satisfy the assumptions in Theorem 5.1 at a point $(\bar{p}, \bar{z})$ with $f(\bar{p}, \bar{z})=0$. Then the condition

$$
\operatorname{rank}\left(\nabla_{z} f(\bar{p}, \bar{z})\right)=m \leq n
$$

is sufficient for the implicit multifunction (5.22) to be pseudo-Lipschitzian around $(\bar{p}, \bar{z})$.

(ii) Condition (5.23) is necessary and sufficient for the pseudo-Lipschitzian property of (5.22) around $(\bar{p}, \bar{z})$ in the following two cases:

(a) $f$ is strictly differentiable in $(p, z)$ at the point $(\bar{p}, \bar{z})$ and $\operatorname{rank}\left(\nabla_{p} f(\bar{p}, \bar{z})\right)=m \leq k$

(b) $f$ admits representation (5.16) and satisfies hypothesis $(\mathrm{H} 3)$ in Theorem 5.8 .

(iii) If $f$ admits representation (5.16) and satisfies hypothesis $(\mathrm{H} 4)$ in Theorem 5.8, then condition (5.23) is necessary and sufficient for the implicit multifunction (5.22) to be pseudo-Lipschitzian around any point $(p, \bar{z})$ with $h(p)=-b(\bar{z})$.

Proof. Assertion (i) follows directly from Theorem 5.1 when $Q(z) \equiv\{0\}$. In case (a) assertion (ii) is implied by assertion (i) in Theorem 5.8 under hypotheses $(\mathrm{H} 1)$ and (H2). In case (b) this assertion follows from (i) in Theorem 5.8 under hypothesis (H3). Assertion (iii) follows from (ii) in Theorem 5.8 where $\bar{P}=\left\{p \in \mathbb{R}^{k}: h(p)=-b(\bar{z})\right\}$.

Note that (5.23) can be referred to the classical Ljusternik-Graves surjectivity condition; see [2]. Therefore, the results obtained ensure that this condition is not only sufficient for the pseudo-Lipschitzian property of implicit multifunctions but also necessary for such a property in a broad setting.

\section{NONSMOOTH GENERALIZED EQUATIONS}

In this section we consider the nonlinear generalized equation (5.1) without any assumptions about smoothness of $f$ in the decision variable $z$ or in the parameter $p$. We study the pseudo-Lipschitzian property of the solution map (5.2) for such a class of nonsmooth generalized equations where $f$ admits a strong approximation in the sense of Robinson [31]. This assumption allows us to develop the procedure from the previous section and obtain effective sufficient conditions for the Lipschitzian stability of (5.1) in terms of the coderivative (2.5) for $f$ and $Q$. 
Following [31], we say that the function $g: \mathbb{R}^{n} \rightarrow \mathbb{R}^{m}$ strongly approximates $f$ in $z$ at $(\bar{p}, \bar{z})$ if for each $\varepsilon>0$ there exist neighborhoods $U$ of $\bar{z}$ and $V$ of $\bar{p}$ such that whenever $z, z^{\prime} \in U$, and $p \in V$ one has

$$
\left\|[f(p, z)-g(z)]-\left[f\left(p, z^{\prime}\right)-g\left(z^{\prime}\right)\right]\right\| \leq \varepsilon\left\|z-z^{\prime}\right\| .
$$

This definition actually means that the difference $f(p, z)-g(z)$ is strictly differentiable in $z$ at $(\bar{p}, \bar{z})$ with the derivative of zero, though neither $f$ nor $g$ is assumed to be differentiable in any sense. Moreover, if $g_{1}$ strongly approximates $f$, then the function $g_{1}+g_{2}$ has the same property for any $g_{2}: \mathbb{R}^{n} \rightarrow \mathbb{R}^{m}$ strictly differentiable at $\bar{z}$ with $\nabla g_{2}(\bar{z})=0$.

Let us observe that any function $f$ in the separable form (5.16) admits a strong approximation in $z$ at any $(\bar{p}, \bar{z})$. Indeed, such a strong approximation is provided by the function $g(z):=b(z)$ which satisfies $(6.1)$ with $\varepsilon=0$.

If $g$ strongly approximates $f$ in $z$ at $(\bar{p}, \bar{z})$, then employing Corollary 2.7 to the difference

$$
\gamma(z):=f(\bar{p}, z)-g(z)
$$

with $\nabla \gamma(\bar{z})=0$, we obtain

$$
D_{z}^{*} f(\bar{p}, \bar{z})\left(y^{*}\right)=D^{*} g(\bar{z})\left(u^{*}\right) \quad \forall y^{*} \in \mathbb{R}^{m}
$$

for any strong approximation $g$, where $D_{z}^{*} f(\bar{p}, \bar{z})$ means the coderivative (2.5) of the vector function $z \rightarrow f(\bar{p}, z)$ at $\bar{z}$. If $f(\bar{p}, \cdot)$ is Lipschitz continuous around $\bar{z}$ and $\partial_{\bar{z}}^{-}\left\langle y^{*}, f\right\rangle(\bar{p}, \bar{z})$ means the subdifferential (2.13) of the function $z \rightarrow\left\langle y^{*}, f(\bar{p}, z)\right\rangle$ at $\bar{z}$, then (6.2) implies

$$
\partial_{z}^{-}\left\langle y^{*}, f\right\rangle(\bar{p}, \bar{z})=\partial^{-}\left\langle y^{*}, g\right\rangle(\bar{z}) \quad \forall y^{*} \in \mathbb{R}^{m}
$$

due to Proposition 2.8. Now using Corollary 2.9, one easily has

$$
\partial_{z}^{-}\left\langle y^{*}, f\right\rangle(\bar{p}, \bar{z}) \subset\left(J_{z} f(\bar{p}, \bar{z})\right)^{*} y^{*}=(J g(\bar{z}))^{*} y^{*} \quad \forall y^{*} \in \mathbb{R}^{m}
$$

where $J_{z} f(\bar{p}, \bar{z})$ means the generalized Jacobian of the vector function $z \rightarrow$ $f(\bar{p}, z)$ at $\bar{z}$.

Let $f$ be strictly differentiable in $z$ at $(\bar{p}, \bar{z})$. Using (6.1) and the definition of strict derivative, one can easily see that the function

$$
g(z):=f(\bar{p}, \bar{z})+\nabla_{z} f(\bar{p}, \bar{z})(z-\bar{z})
$$

strongly approximates $f$ in $z$ at $(\bar{p}, \bar{z})$. Note that there are other strong approximations $g$ of $f$ in $z$ at this point but all of them are strictly differentiable at $\bar{z}$ with

$$
\nabla g(\bar{z})=\nabla_{z} f(\bar{p}, \bar{z})
$$

It is easy to get examples where nonsmooth functions $f$ admit strong approximations which must also be nonsmooth. In particular, if $f$ is strictly differentiable in $z$ at $(\bar{p}, \bar{z})$ and $\theta: \mathbb{R}^{r} \rightarrow \mathbb{R}^{n}$ is locally Lipschitzian around $\bar{w}$ with $\theta(\bar{w})=\bar{z}$, then the composite function

$$
\psi(p, w):=f(p, \theta(w))
$$

has a strong approximation in $w$ at $(\bar{p}, \bar{w})$; $\mathrm{cf}$. [31, Proposition 2.5]. If $f$ has the so-called strong partial B-derivative $D_{z} f(\bar{p}, \bar{z})$ in $z$ at $(\bar{p}, \bar{z})$, then the function

$$
f(\bar{p}, \bar{z})+D_{z} f(\bar{p}, \bar{z})(z-\bar{z})
$$


provides a strong approximation of $f$ in $z$ at $(\bar{p}, \bar{z})$; see $[10,26,31]$ for more details.

The following result ensures a general sufficient condition for the pseudoLipschitzian property of the solution set to the nonsmooth generalized equation (5.1).

Theorem 6.1. Let $f$ be locally Lipschitzian in $p$ uniformly in $z$ around the point $(\bar{p}, \bar{z})$ satisfying $(5.1)$ and let $f$ admit a strong approximation in $z$ at $(\bar{p}, \bar{z})$. Assume that either $f(\bar{p}, \cdot)$ is Lipschitz continuous around $\bar{z}$ or $Q$ is pseudo-Lipschitzian around $(\bar{z},-f(\bar{p}, \bar{z}))$, i.e.,

$$
D^{*} Q(\bar{z},-f(\bar{p}, \bar{z}))(0)=\{0\} .
$$

Then the condition

$$
\left[0 \in D_{z}^{*} f(\bar{p}, \bar{z})\left(y^{*}\right)+D^{*} Q(\bar{z},-f(\bar{p}, \bar{z}))\left(y^{*}\right)\right] \Rightarrow y^{*}=0
$$

is sufficient for the solution map (5.2) to be pseudo-Lipschitzian around $(\bar{p}, \bar{z})$. Proof. Let $g$ be some strong approximation of $f$ in $z$ at $(\bar{p}, \bar{z})$ which exists according to the assumptions. Note that $g$ has to be Lipschitz continuous around $\bar{z}$ if $f(\bar{p}, \cdot)$ possesses this property. Using this strong approximation, we consider the approximate generalized equation

$$
0 \in g(z)-y+Q(z)
$$

and its solution map

$$
\Gamma(y):=\left\{z \in \mathbb{R}^{n}: 0 \in g(z)-y+Q(z)\right\}
$$

depending on the linear parameter $y \in \mathbb{R}^{m}$. Denoting $\bar{y}=g(\bar{z})-f(\bar{p}, \bar{z})$, one can see that $(\bar{y}, \bar{z})$ satisfies the approximate generalized equation (6.8). According to Dontchev and Hager [6, Theorem 4.1], the pseudo-Lipschitzian property of $(6.9)$ around $(\bar{y}, \bar{z})$ implies, under the assumptions made, the same property of the solution map (5.2) around $(\bar{p}, \bar{z})$. It is obvious that for each $y$ the set $\Gamma(y)$ in (6.9) coincides with the value of the inverse mapping $L^{-1}(y)$ to the multifunction

$$
L(z):=g(z)+Q(z) .
$$

Therefore, the pseudo-Lipschitzian property of $\Gamma$ around $(\bar{y}, \bar{z})$ actually means that multifunction (6.10) has the pseudo-Lipschitzian inverse around this point. By virtue of Corollary 3.8(i), it is equivalent to the condition

$$
\left[0 \in D^{*} L(\bar{z}, \bar{y})\left(y^{*}\right)\right] \Rightarrow y^{*}=0
$$

in terms of the coderivative (2.5) for multifunction (6.10). Now using Corollary 3.6 for representing the coderivative of the sum in $(6.10)$, one can see that, under the assumptions made, $(6.11)$ is automatically fulfilled if

$$
\left[0 \in D^{*} g(\bar{z})\left(y^{*}\right)+D^{*} Q(\bar{z},-f(\bar{p}, \bar{z}))\left(y^{*}\right)\right] \Rightarrow y^{*}=0 .
$$

But due to (6.2), the latter condition coincides with (6.7) which is, therefore, a sufficient condition for the solution map (5.2) to be pseudo-Lipschitzian around $(\bar{p}, \bar{z})$.

Remark 6.2. If $f$ is strictly differentiable in $z$ at the point $(\bar{p}, \bar{z})$, then it always admits the strong approximation (6.5). Taking this approximation in 
Theorem 6.1, we can see that the result obtained implies Theorem 5.1 in the previous section. Let us observe that any other strong approximation $g$ of such a function $f$ provides the same result in Theorem 6.1 because one always has property (6.6). The advantage of the smooth case in $\S 5$ consists in proving the necessity of the sufficient condition obtained for the solution set (5.2) being pseudo-Lipschitzian around $(\bar{p}, \bar{z})$ under some additional assumptions. Using the procedure above, we cannot obtain any results in this direction without the strict differentiability property of $f$ in $z$ at $(\bar{p}, \bar{z})$.

Let us formulate a corollary of Theorem 6.1 for the case of separable functions (5.16) in the nonsmooth generalized equation (5.1) where the main assumption of the theorem is automatically fulfilled.

Corollary 6.3. Let $(\bar{p}, \bar{z})$ satisfy the separable generalized equation

$$
0 \in b(z)+h(p)+Q(z)
$$

where both $b$ and $h$ are Lipschitz continuous around $\bar{z}$ and $\bar{p}$ respectively. Then the condition

$$
\left[0 \in \partial^{-}\left\langle y^{*}, b\right\rangle(\bar{z})+D^{*} Q(\bar{z},-b(\bar{z})-h(\bar{p}))\left(y^{*}\right)\right] \Rightarrow y^{*}=0
$$

is sufficient for the solution map (6.12) to be pseudo-Lipschitzian around $(\bar{p}, \bar{z})$. Proof. It is obvious that the function $f(p, z):=b(z)+h(p)$ satisfies all the assumptions in Theorem 6.1 with the strong approximation $g(z):=b(z)$. For this $f$, condition (6.7) is reduced to (6.13).

Now we consider some corollaries of the Theorem 6.1 for the case of Lipschitzian functions $f$. One can immediately obtain their reformulations in the separable case (6.12). The following result is expressed in terms of the partial generalized Jacobian of $f$ in $z$.

Corollary 6.4. Let $f$ satisfy all the assumptions in Theorem 6.1. Then the condition

$$
\left[0 \in\left(J_{z} f(\bar{p}, \bar{z})\right)^{*} y^{*}+D^{*} Q(\bar{z},-f(\bar{p}, \bar{z}))\left(y^{*}\right)\right] \Rightarrow y^{*}=0
$$

ensures the pseudo-Lipschitzian property of the solution map (5.2) around $(\bar{p}, \bar{z})$. Proof. This follows from Theorem 6.1 by virtue of the inclusion in formula (6.4).

Let us obtain some effective concretizations of the results in Theorem 6.1 and Corollary 6.4 for multifunctions $Q$ of special structure.

Corollary 6.5. Let the multifunction $Q$ in (5.1) have convex graph and let the function $f$ satisfy all the assumptions in Theorem 6.1. For any matrix $A \in$ $J_{z} f(\bar{p}, \bar{z})$ we consider the multivalued operator

$$
L_{A}:=f(\bar{p}, \bar{z})+A(z-\bar{z})+Q(z)
$$

and assume that

$$
0 \in \operatorname{int}\left(\operatorname{Im} L_{A}\right) \quad \forall A \in J_{z} f(\bar{p}, \bar{z}) .
$$

Then the solution map (5.2) is pseudo-Lipschitzian around $(\bar{p}, \bar{z})$.

Proof. Considering (6.14), we take any matrix $A \in J_{z} f(\bar{p}, \bar{z})$ such that

$$
0 \in A^{*} y^{*}+D^{*} Q(\bar{z},-f(\bar{p}, \bar{z}))\left(y^{*}\right) .
$$


Using Proposition 2.5(i) for the representation of the coderivative for the convexgraph multifunction $Q$ and taking into account that

$$
\left\langle A^{*} y^{*}, z-\bar{z}\right\rangle=\left\langle y^{*}, A(z-\bar{z})\right\rangle,
$$

one can rewrite (6.16) in the equivalent form

$$
\left\langle y^{*}, u\right\rangle \geq 0 \text { for any } u \in \operatorname{Im} L_{A} .
$$

Similarly to the proof of Corollary 4.2, now we conclude that condition (6.15) is necessary and sufficient for the adjoint generalized equation (6.16) having the only trivial solution $y^{*}=0$ when $A$ is an arbitrary matrix belonging to $J_{z} f(\bar{p}, \bar{z})$. Therefore, (6.15) implies (6.14) and provides a sufficient condition for the pseudo-Lipschitzian property of the solution map to the nonsmooth generalized equation (5.1) with a convex-graph multifunction $Q$.

Corollary 6.6. Let $f$ satisfy all the assumptions in Theorem 6.1 and let $Q$ be represented in form (4.9) with some closed and convex sets $E$ and $\Omega$. Then the condition

$$
0 \in \operatorname{int}\{f(\bar{p}, \bar{z})+A(\Omega-\bar{z})+E\} \quad \forall A \in J_{z} f(\bar{p}, \bar{z})
$$

is sufficient for the solution map (5.2) to be pseudo-Lipschitzian around $(\bar{p}, \bar{z})$.

Proof. This follows directly from Corollary 6.4 .

Corollary 6.7. Let $f$ satisfy all the assumptions in Theorem 6.1 and let $Q$ be represented in form (4.11) where $\Lambda \subset \mathbb{R}^{q}$ is a closed set and $\theta: \mathbb{R}^{n} \times$ $\mathbb{R}^{m} \rightarrow \mathbb{R}^{q}$ is a vector function continuous around the point $\left.(\bar{z})-f(\bar{p}, \bar{z})\right)$ with $\theta(\bar{z},-f(\bar{p}, \bar{z})) \in \Lambda$. Then:

(i) The condition

$$
\begin{gathered}
{\left[(0,0) \in\left(\partial_{z}^{-}\left\langle y^{*}, f\right\rangle(\bar{p}, \bar{z}), y^{*}\right)+D^{*} \theta(\bar{z},-f(\bar{p}, \bar{z}))\left(w^{*}\right) \&\right.} \\
\left.w^{*} \in N(\theta(\bar{z},-f(\bar{p}, \bar{z})) \mid \Lambda)\right] \Rightarrow w^{*}=0 \& y^{*}=0
\end{gathered}
$$

is sufficient for the solution map (5.2) to be pseudo-Lipschitzian around $(\bar{p}, \bar{z})$.

(ii) If $\theta$ is Lipschitz continuous around $(\bar{z},-f(\bar{p}, \bar{z}))$, then the sufficient condition (6.17) is equivalent to

$$
\begin{gathered}
{\left[(0,0) \in\left(\partial_{z}^{-}\left\langle y^{*}, f\right\rangle(\bar{p}, \bar{z}), y^{*}\right)+\partial^{-}\left\langle w^{*}, \theta\right\rangle(\bar{z},-f(\bar{p}, \bar{z})) \&\right.} \\
\left.w^{*} \in N(\theta(\bar{z},-f(\bar{p}, \bar{z})) \mid \Lambda)\right] \Rightarrow w^{*}=0
\end{gathered}
$$

which is automatically fulfilled when

$$
\begin{array}{r}
{\left[(0,0) \in\left(\left(J_{z} f(\bar{p}, \bar{z})\right)^{*} y^{*}, y^{*}\right)+(J \theta(\bar{z},-f(\bar{p}, \bar{z})))^{*} w^{*} \&\right.} \\
\left.w^{*} \in N(\theta(\bar{z},-f(\bar{p}, \bar{z})) \mid \Lambda)\right] \Rightarrow w^{*}=0 .
\end{array}
$$

(iii) If $\theta$ is strictly differentiable at $(\bar{z},-f(\bar{p}, \bar{z}))$, then the sufficient condition (6.19) is equivalent to

$$
\begin{gathered}
{\left[0 \in\left(\nabla_{y} \theta(\bar{z},-f(\bar{p}, \bar{z})) J_{z} f(\bar{p}, \bar{z})-\nabla_{z} \theta(\bar{z},-f(\bar{p}, \bar{z}))\right)^{*} w^{*} \&\right.} \\
\left.w^{*} \in N(\theta(\bar{z},-f(\bar{p}, \bar{z})) \mid \Lambda)\right] \Rightarrow w^{*}=0
\end{gathered}
$$

which is automatically fulfilled when

$$
\operatorname{rank}\left(\nabla_{y} \theta(\bar{z},-f(\bar{p}, \bar{z})) J_{z} f(\bar{p}, \bar{z})-\nabla_{z} \theta(\bar{z},-f(\bar{p}, \bar{z}))\right)=q \leq n .
$$


Proof. First we prove (i). Let us obtain a representation of the coderivative (2.5) for the multifunction $Q$ of the form (4.11). Using the definition of the coderivative and Corollary 2.11 , we have the inclusion

$$
\begin{aligned}
& D^{*} Q(\bar{z},-f(\bar{p}, \bar{z}))\left(y^{*}\right) \\
& \quad \subset\left\{z^{*} \in \mathbb{R}^{n}:\left(z^{*},-y^{*}\right) \in D^{*} \theta(\bar{z},-f(\bar{p}, \bar{z}))\left(w^{*}\right)\right. \\
& \text { with } \left.w^{*} \in N(\theta(\bar{z},-f(\bar{p}, \bar{z})) \mid \Lambda)\right\}
\end{aligned}
$$

under the condition

$$
\operatorname{Ker} D^{*} \theta(\bar{z},-f(\bar{p}, \bar{z})) \cap N(\theta(\bar{z},-f(\bar{p}, \bar{z})) \mid \Lambda)=\{0\} .
$$

Now one can easily see that if (6.17) holds, then (6.23) also holds and hence (6.17) implies (6.7) by virtue of representation (6.22). This proves assertion (i) in the corollary under consideration.

Under the Lipschitzian assumption in (ii), the equivalence between (6.17) and (6.18) follows from Proposition 2.8. Condition (6.19) always implies (6.18) due to the inclusion in formula (6.4).

If $\theta$ is strictly differentiable at $(\bar{z},-f(\bar{p}, \bar{z}))$, then

$$
J \theta(\bar{z},-f(\bar{p}, \bar{z}))=\left(\nabla_{z} \theta(\bar{z},-f(\bar{p}, \bar{z})), \nabla_{y} \theta(\bar{z},-f(\bar{p}, \bar{z}))\right)
$$

and (6.19) is reduced to (6.20) by elementary transformations. It is obvious that the rank condition (6.20) implies $w^{*}=0$ due to the first part of (6.20).

The results obtained in Corollary 6.7 generalize the corresponding results in Corollaries 4.5, 5.5, and the sufficiency part of Corollary 4.4. Now we consider a special case of Corollary 6.7 where $Q$ admits the classical representation (4.21) with real-valued functions $\theta_{i}$ strictly differentiable at $(\bar{z},-f(\bar{p}, \bar{z}))$. Using notation (4.22), we define the family of matrices

$$
C_{A}:=\nabla_{y} \theta(\bar{z},-f(\bar{p}, \bar{z})) A-\nabla_{z} \theta(\bar{z},-f(\bar{p}, \bar{z}))
$$

depending on the matrix parameter $A$. The following result provides a sufficient condition for the Lipschitzian stability of the nonsmooth system (5.1), (4.21) in a generalized form of the Mangasarian-Fromovitz constraint qualification.

Corollary 6.8. Let $f$ satisfy all the assumptions in Theorem 6.1 and let $Q$ be represented in the form (4.21) where the functions $\theta_{i}$ are strictly differentiable at $(\bar{z},-f(\bar{p}, \bar{z}))$ for all $i=1,2, \ldots, q$. Assume that for any $A \in J_{z}(\bar{p}, \bar{z})$ the qualification condition (5.14) is fulfilled where $\left(c_{1}, c_{2}, \ldots, c_{q}\right)$ are the vector columns of the matrix $C_{A}$ in (6.24). Then the solution map (5.2) to (5.1), (4.21) is pseudo-Lipschitzian around $(\bar{p}, \bar{z})$.

Proof. This follows from assertion (iii) in Corollary 6.7 for the case of $\Lambda$ having the form (4.24).

Now let us consider a general setting when the multifunction $Q$ is represented in the subdifferential form (4.25) which covers the case of nonsmooth variational inequalities. In this setting, Theorem 6.1 implies the following nonsmooth extensions of the corresponding results in Corollaries 4.8 and 5.6.

Corollary 6.9. Let $f$ satisfy all the assumptions in Theorem 6.1 and let $Q$ be the subdifferential mapping (4.25) generated by an extended-real-valued function 
$\varphi$. Then the solution map (5.2) is pseudo-Lipschitzian around $(\bar{p}, \bar{z})$ if the relationship

$$
\left[0 \in \partial_{z}^{-}\left\langle y^{*}, f\right\rangle(\bar{p}, \bar{z})+\partial^{2,-} \varphi(\bar{z},-f(\bar{p}, \bar{z}))\left(y^{*}\right)\right] \Rightarrow y^{*}=0
$$

holds. It happens, in particular, when one has

$$
\left[0 \in\left(J_{z} f(\bar{p}, \bar{z})\right)^{*} y^{*}+\partial^{2,-} \varphi(\bar{z},-f(\bar{p}, \bar{z}))\left(y^{*}\right)\right] \Rightarrow y^{*}=0 .
$$

Proof. This follows from Theorem 6.1 and Corollary 6.4 due to the definition of the second order subdifferential (2.14).

Remark 6.10. Let us compare the conditions obtained in Theorem 6.1 and its corollaries with the corresponding results in Mordukhovich [23]. It is proved in [23, Corollary 5.2] that if $f$ is Lipschitz continuous in both variables $(p, z)$ around $(\bar{p}, \bar{z})$ and $\operatorname{pr}_{z} \partial^{-}\left\langle y^{*}, f\right\rangle(\bar{p}, \bar{z})$ denotes the projection of the subdifferential $\partial^{-}\left\langle y^{*}, f\right\rangle(\bar{p}, \bar{z}) \subset \mathbb{R}^{k} \times \mathbb{R}^{n}$ on the space $\mathbb{R}^{n}$, then the condition

$$
\left[0 \in \operatorname{pr}_{z} \partial^{-}\left\langle y^{*}, f\right\rangle(\bar{p}, \bar{z})+D^{*} Q(\bar{z},-f(\bar{p}, \bar{z}))\left(y^{*}\right)\right] \Rightarrow y^{*}=0
$$

is sufficient for the solution map (5.2) being pseudo-Lipschitzian around $(\bar{p}, \bar{z})$. This condition is satisfied a fortiori when

$$
\left[0 \in\left(\operatorname{pr}_{z} J f(\bar{p}, \bar{z})\right)^{*} y^{*}+D^{*} Q(\bar{z},-f(\bar{p}, \bar{z}))\left(y^{*}\right)\right] \Rightarrow y^{*}=0
$$

in terms of the projection of the generalized Jacobian $J f(\bar{p}, \bar{z}) \subset \mathbb{R}^{m k} \times \mathbb{R}^{m n}$ on $\mathbb{R}^{m n}$; see [23, Corollary 5.3]. By virtue of Proposition 2.8, the sufficient condition (6.7) in Theorem 6.1 is equivalent to

$$
\left[0 \in \partial_{z}^{-}\left\langle y^{*}, f\right\rangle(\bar{p}, \bar{z})\left(y^{*}\right)+D^{*} Q(\bar{z},-f(\bar{p}, \bar{z}))\left(y^{*}\right)\right] \Rightarrow y^{*}=0
$$

in the case of Lipschitzian functions $f$. Now employing Corollaries 2.13 and 2.14 , one can conclude that the sufficient conditions in Theorem 6.1 and Corollary $6.4 \mathrm{imply,} \mathrm{respectively,} \mathrm{(6.27)} \mathrm{and} \mathrm{(6.28).} \mathrm{Moreover,} \mathrm{these} \mathrm{two} \mathrm{pairs} \mathrm{of}$ conditions coincide for the separable generalized equations (6.12); it follows from the equality case in Corollaries 2.13 and 2.14. Observe, however, that the conditions in [23] are proved without any assumptions about the existence of a strong approximation of $f$ in $z$. Therefore, the results in Theorem 6.1 and Corollary 6.4 strengthen the corresponding results in [23] when $f$ admits a strong approximation in $z$ at $(\bar{p}, \bar{z})$ (in particular, for smooth generalized equations in $\S 5)$. In a general nonsmooth setting, these two groups of results are independent. Note that in [23, Theorem 5.1] one can find a sufficient condition for the solution map (5.2) to be pseudo-Lipschitzian around $(\bar{p}, \bar{z})$ in the case when $f$ is merely continuous in $(p, z)$ around this point. This condition is expressed in terms of the coderivative of $f$ in both $(p, z)$ having a form different from (6.7), (6.27), and (6.29); cf. condition (7.3).

Remark 6.11. Following the schemes in proving Corollaries 6.5-6.8, one can obtain the corresponding corollaries of the sufficient conditions (6.27) and (6.28) for the special classes of multifunctions $Q$. In these results, the sets $\partial_{z}^{-}\left\langle y^{*}, f\right\rangle(\bar{p}, \bar{z})$ and $J_{z} f(\bar{p}, \bar{z})$ are changed, to $\operatorname{pr}_{z} \partial^{-}\left\langle y^{*}, f\right\rangle(\bar{p}, \bar{z})$ and $\operatorname{pr}_{z} J f(\bar{p}, \bar{z})$, respectively.

To conclude this section, we consider a special case of Theorem 6.1 with $Q(z) \equiv\{0\}$. In this situation, the solution map (5.2) is reduced to the implicit multifunction (5.22) defined by the equation $f(p, z)=0$ with a nonsmooth function $f$. 
Corollary 6.12. Let $f$ be locally Lipschitzian in $p$ uniformly in $z$ around the point $(\bar{p}, \bar{z})$ with $f(\bar{p}, \bar{z})=0$ and let $f$ admit a strong approximation in $z$ at $(\bar{p}, \bar{z})$. Then the implicit multifunction (5.22) is pseudo-Lipschitzian around $(\bar{p}, \bar{z})$ if

$$
\operatorname{Ker} D_{z}^{*} f(\bar{p}, \bar{z})=\{0\} .
$$

If $f(\bar{p}, \cdot)$ is Lipschitz continuous around $\bar{z}$, then $(6.30)$ is equivalent to

$$
\operatorname{Ker} \partial_{z}^{-}\langle\cdot, f\rangle(\bar{p}, \bar{z})=\{0\}
$$

which is automatically fulfilled when

$$
\operatorname{rank}\left(J_{z} f(\bar{p}, \bar{z})\right)=m \leq n .
$$

Proof. This follows directly from Theorem 6.1 and Corollary 6.4 when $Q(z) \equiv$ $\{0\}$.

Remark 6.13. Condition (6.31) always implies that

$$
\left[0 \in \operatorname{pr}_{z} \partial^{-}\left\langle y^{*}, f\right\rangle(\bar{p}, \bar{z})\right] \Rightarrow y^{*}=0 .
$$

In Mordukhovich [23, Corollary 4.14], the latter condition is proved to be sufficient for the pseudo-Lipschitzness of the implicit multifunction (5.22) when $f$ is Lipschitz continuous in both $(p, z)$ around $(\bar{p}, \bar{z})$ but may not admit a strong approximation in $z$. Moreover, condition (6.32) for such $f$ with $m=n$ ensures not only Lipschitzness but also (locally) single-valuedness of the implicit mapping in (5.22); see Clarke [4, §7]. Note that Kummer [13] obtained a necessary and sufficient condition for the implicit mapping (5.22) to be single-valued and Lipschitz continuous around $\bar{p}$ in the case of Lipschitzian functions $f$. Some other results in this direction are considered in the next section under different assumptions about $f$.

Remark 6.14. We have studied the pseudo-Lipschitzian property of solution maps to generalized equations around the given point $(\bar{p}, \bar{z})$. If the conditions obtained are fulfilled for all points $\bar{z}$ satisfying the corresponding generalized equations together with given $\bar{p}$, then these results provide sufficient conditions (respectively, necessary and sufficient conditions) for the sub-Lipschitzian property of the solution maps around $\bar{p}$. This follows from Proposition 3.4(i). If, in addition, the corresponding solution maps are locally bounded around $\bar{p}$, then the conditions obtained ensure their classical locally Lipschitzian behavior around this point, due to Proposition 3.4(ii).

\section{MONOTONE PROCESSES AND VARIATIONAL INEQUALITIES}

This concluding section is devoted to obtaining effective sufficient conditions ensuring the (locally) single-valuedness and Lipschitz continuity of the solution map to the generalized equation (5.1) around a given point $(\bar{p}, \bar{z})$ satisfying (5.1). We consider a general setting when the continuous function $f: \mathbb{R}^{k} \times \mathbb{R}^{n} \rightarrow \mathbb{R}^{n}$ may be nonsmooth around $(\bar{p}, \bar{z})$ and the closed-graph multifunction $Q$ is a monotone operator from $\mathbb{R}^{n}$ into itself. This includes, in particular, the cases of nonsmooth variational inequalities and complementarity problems. If $f$ happens to be strictly differentiable at $(\bar{p}, \bar{z})$, then the conditions obtained appear to be necessary and sufficient for the locally singlevaluedness and Lipschitz continuity of the solution map (5.2) under some additional assumptions. In this way, we obtain, in particular, a necessary and 
sufficient condition for Robinson's strong regularity property [29] in terms of the coderivative $(2.5)$ of any monotone operator $Q$ in the smooth generalized equation (5.1). The results obtained in this section are based on the previous conditions ensuring the pseudo-Lipschitzian property of the solution map and on the single-valuedness property of monotone operators in Proposition 3.12.

We say that the solution map (5.2) is locally single-valued and Lipschitz continuous around $(\bar{p}, \bar{z})$ if there exist neighborhoods $U$ of $\bar{p}$ and $V$ of $\bar{z}$ as well as a Lipschitzian function $\tilde{z}: U \rightarrow V$ such that $\tilde{z}(p)$ is a unique solution in $V$ to the generalized equation $(5.1)$ for each $p \in U$.

Theorem 7.1. Let $f$ be locally Lipschitzian in $p$ uniformly in $z$ around the point $(\bar{p}, \bar{z})$ satisfying $(5.1)$ and let $f$ admit a strong approximation in $z$ at $(\bar{p}, \bar{z})$ which is either positive or negative monotone simultaneously with the multifunction $Q$. Assume that either $f(\bar{p}, \cdot)$ is Lipschitz continuous around $\bar{z}$ or $Q$ is pseudo-Lipschitzian around $(\bar{z},-f(\bar{p}, \bar{z}))$. Then condition (6.7) is sufficient to the solution map (5.2) to be locally single-valued and Lipschitz continuous around $(\bar{p}, \bar{z})$.

Proof. Let $g$ be any strong approximation of $f$ in $z$ at $(\bar{p}, \bar{z})$ satisfying the assumptions imposed. Considering the sum $g+Q$ of two monotone operators, one can see that this sum is also monotone in the same direction. This easily implies the monotonicity property of the inverse operator $(g+Q)^{-1}$. Now let us consider the solution map (6.9) to the approximate generalized equation (6.8). It is obvious that values $\Gamma(y)$ in (6.9) coincide with values of the inverse operator $(g+Q)^{-1}(y)$. Therefore, the solution map (6.9) is a monotone operator from $\mathbb{R}^{n}$ into itself. According to the proof of Theorem 6.1 , condition (6.7) ensures the pseudo-Lipschitzian property of (6.9) around the point $(g(\bar{z})-f(\bar{p}, \bar{z}), \bar{z})$. Employing Proposition 3.12, we conclude that the monotone operator $\Gamma$ in (6.9) is actually single-valued and Lipschitz continuous around the point $\bar{y}:=$ $g(\bar{z})-f(\bar{p}, \bar{z})$ with $\Gamma(\bar{y})=\{\bar{z}\}$.

Now one can use the results in Robinson [29, 31] and Dontchev and Hager [6] about locally single-valued and Lipschitz continuous solutions to parametric generalized equations via the same properties of solutions to their strong approximations. The most general (to the best of our knowledge) result in this direction obtained in Dontchev and Hager [6, assertion 3 in Theorem 4.1]. They prove that, under the assumptions of Theorem 7.1, locally single-valuedness and Lipschitzness of the map $\Gamma$ in (6.9) around $(\bar{y}, \bar{z})$ imply these properties of the solution map (5.2) around the point $(\bar{p}, \bar{z})$. Therefore, condition (6.7) is sufficient for the "monotone" generalized equation (5.1) having the unique solution around $(\bar{p}, \bar{z})$ with the Lipschitzian dependence on the parameter.

Corollary 7.2. Let all the assumptions in Theorem 7.1 be fulfilled with $f(\bar{p}, \cdot)$ being Lipschitz continuous around $\bar{z}$. Then condition (6.14), stated in terms of the partial generalized Jacobian of $f$ in $z$, is sufficient for locally uniqueness and Lipschitz continuity of the solution map to the generalized equation (5.1) around $(\bar{p}, \bar{z})$.

Proof. This follows from Theorem 7.1 because (6.14) always implies (6.7) due to the inclusion in formula (6.4).

Now we obtain two corollaries of Theorem 7.1 for some cases when $f$ automatically admits strong approximations in $z$. 
Corollary 7.3. Let $f$ be strictly differentiable in $z$ at $(\bar{p}, \bar{z})$ and let it be locally Lipschitzian in $p$ uniformly in $z$ around $(\bar{p}, \bar{z})$. Assume that $Q$ is positive (respectively, negative) monotone and the matrix $\nabla_{z} f(\bar{p}, \bar{z})$ is positive (negative) semidefinite with respect to $\operatorname{Dom} Q$ in the sense that

$$
\left\langle\nabla_{z} f(\bar{p}, \bar{z}) u, u\right\rangle \geq 0(\leq 0) \quad \text { for all } u \in \operatorname{Dom} Q-\operatorname{Dom} Q .
$$

Then condition (5.7) is sufficient for the solution map (5.2) to be locally singlevalued and Lipschitz continuous around $(\bar{p}, \bar{z})$.

Proof. If $f$ is strictly differentiable in $z$ at $(\bar{p}, \bar{z})$, then the function $g$ in (6.5) provides a strong approximation of $f$ in $z$ at this point. One can easily see that the single-valued operator $z \rightarrow f(\bar{p}, \bar{z})+\nabla_{z} f(\bar{p}, \bar{z})(z-\bar{z})$ from $\mathbb{R}^{n}$ into itself is positive (negative) monotone on the domain of $Q$ if the matrix $\nabla_{z} f(\bar{p}, \bar{z})$ is positive (respectively, negative) semidefinite with respect to this set. Therefore, we meet all the assumptions in Theorem 7.1 where condition (6.7) is reduced to (5.7) in the smooth case under consideration.

Corollary 7.4. Let $(\bar{p}, \bar{z})$ satisfy the separable generalized equation (6.12) where both $b$ and $h$ are Lipschitz continuous around $\bar{z}$ and $\bar{p}$ respectively. If $b$ is positive or negative monotone simultaneously with the multifunction $Q$, then condition (6.13) is sufficient for the solution map

$$
\Sigma(p):=\left\{z \in \mathbb{R}^{n}: 0 \in b(z)+h(p)+Q(z)\right\}
$$

to (6.12) being locally single-valued and Lipschitz continuous around $(\bar{p}, \bar{z})$.

Proof. We meet all the assumptions in Theorem 7.1 with the function $g(z)=$ $b(z)$ providing a strong approximation of $f$ in $z$ at $(\bar{p}, \bar{z})$. Condition (6.7) coincides with (6.13) for this case.

Following the procedure in $\S \S 5$ and 6 , one can formulate the corresponding corollaries of Theorem 7.1 and Corollaries 7.2-7.4 for the cases when $Q$ admits one of the special structures considered above. Now we obtain a new result for the separable generalized equation (6.12) with an arbitrary multifunction $Q$ and a function $b$ being merely continuous around $\bar{z}$.

Theorem 7.5. Let $(\bar{p}, \bar{z})$ satisfy the separable generalized equation (6.12) where $b$ is continuous around $\bar{z}$ and $h$ is Lipschitz continuous around $\bar{p}$. If $b$ is either positive or negative monotone simultaneously with the multifunction $Q$, then the condition

$$
\left[z^{*} \in\left(-D^{*} b(\bar{z})\left(y^{*}\right)\right) \cap D^{*} Q(\bar{z},-b(\bar{z})-h(\bar{p}))\left(y^{*}\right)\right] \Rightarrow y^{*}=0 \& z^{*}=0
$$

is sufficient for the solution map (7.1) being locally single-valued and Lipschitz continuous around $(\bar{p}, \bar{z})$.

Proof. According to Mordukhovich [23, Theorem 5.1], the condition

$$
\begin{aligned}
{\left[\left(p^{*},-z^{*}\right)\right.} & \left.\in D^{*}(b+h)(\bar{p}, \bar{z})\left(y^{*}\right) \& z^{*} \in Q(\bar{z},-b(\bar{z})-h(\bar{p}))\left(y^{*}\right)\right] \\
\Rightarrow p^{*} & =0, \quad z^{*}=0 \& y^{*}=0
\end{aligned}
$$

ensures the pseudo-Lipschitzian property of the solution map (7.1) around $(\bar{p}, \bar{z})$ under the general assumptions in the theorem. One can easily see that

$$
D^{*} b(\bar{p}, \bar{z})\left(y^{*}\right)=\left(0, D^{*} b(\bar{z})\left(y^{*}\right)\right) \text { and } D^{*} h(\bar{p}, \bar{z})\left(y^{*}\right)=\left(D^{*} h(\bar{p})\left(y^{*}\right), 0\right) \text {. }
$$


Now employing Corollary 3.6 to the sum $b+h$ where $h$ is Lipschitzian, we obtain

$$
D^{*}(b+h)(\bar{p}, \bar{z})\left(y^{*}\right) \subset\left(\partial^{-}\left\langle y^{*}, h\right\rangle(\bar{p}), D^{*} b(\bar{z})\left(y^{*}\right)\right) .
$$

Therefore, condition (7.2) implies (7.3), thus ensuring the pseudo-Lipschitzian behavior of the solution map (7.1) around $(\bar{p}, \bar{z})$. It is clear that this condition is also sufficient for the pseudo-Lipschitzian property of the multifunction

$$
\Gamma(v):=\left\{z \in \mathbb{R}^{n}: 0 \in b(z)-v+Q(z)\right\}
$$

around $(h(\bar{p}), \bar{z})$. The latter multifunction is the solution map to the auxiliary generalized equation $0 \in b(z)-v+Q(z)$ with the linear additive parameter $v \in$ $\mathbb{R}^{n}$. Under the monotonicity assumptions made in the theorem, the operator $(b+Q)(z)$ is monotone. This implies the monotonicity of the multifunction $\Gamma(v)=(b+Q)^{-1}(v)$. Now employing Proposition 3.12, we can conclude that condition (7.2) actually ensures the single-valuedness and Lipschitz continuity of $\Gamma$ around $v=h(\bar{p})$ with $\Gamma(\bar{v})=\{\bar{z}\}$. Therefore, the solution map (7.1) represented in form $\Sigma(p)=\Gamma(h(p))$ turns out to be locally single-valued and Lipschitz continuous around $(\bar{p}, \bar{z})$.

Remark 7.6. Let us observe that condition (7.2) is equivalent to

$$
\left[0 \in D^{*} b(\bar{z})\left(y^{*}\right)+D^{*} Q(\bar{z},-b(\bar{z})-h(\bar{p}))\left(y^{*}\right)\right] \Rightarrow y^{*}=0
$$

if either the multifunction $Q$ is pseudo-Lipschitzian around $(\bar{z},-b(\bar{z})-h(\bar{p}))$ or the function $b$ is Lipschitz continuous around $\bar{z}$. This follows from Proposition 3.5. Note that (7.5) is reduced to (6.13) in the case of $b$ being Lipschitz continuous around $\bar{z}$. Therefore, Theorem 7.5 generalizes Corollary 7.4 obtained above from Theorem 7.1.

Now using the representations of the coderivative $D^{*} Q$ form Proposition 2.5(i) and Corollary 2.11 for multifunctions $Q$ of special structure, one can easily obtain the corresponding concretizations of Theorem 7.5; cf. Corollaries 6.5-6.8. Let us formulate a result for the case of representation (4.9) where $E$ and $\Omega$ may be nonconvex sets belonging to orthogonal affine subspaces in $\mathbb{R}^{n}$.

Corollary 7.7. Let $(\bar{p}, \bar{z})$ satisfy the separable generalized equation (6.12) where $h$ is Lipschitz continuous around $\bar{p}$ and $b$ is a monotone mapping continuous around $\bar{z}$. Suppose that the multifunction $Q$ admits representation (4.9) where $E \subset \mathbb{R}^{n}$ and $\Omega \subset \mathbb{R}^{n}$ are closed sets such that $E-E \subset L$ and $\Omega-\Omega \subset L^{\perp}$ for some linear subspace $L$ in $\mathbb{R}^{n}$. Then the condition

$$
\begin{aligned}
{\left[0 \in z^{*}+D^{*} b(\bar{z})\left(y^{*}\right) \text { with } z^{*}\right.} & \in N(\bar{z} \mid \Omega) \text { and } \\
-y^{*} & \in N(-b(\bar{z})-h(\bar{p}) \mid E)] \Rightarrow z^{*}=0 \& y^{*}=0
\end{aligned}
$$

is sufficient for the solution map (7.1) to be locally single-valued and Lipschitz continuous around $(\bar{p}, \bar{z})$.

Proof. First let us observe that the normal cone (2.1) enjoys the multiplicative property

$$
N\left(\left(\bar{x}_{1}, \bar{x}_{2}\right) \mid \Omega_{1} \times \Omega_{2}\right)=N\left(\bar{x}_{1} \mid \Omega_{1}\right) \times N\left(\bar{x}_{2} \mid \Omega_{2}\right)
$$

for any closed sets $\Omega_{1} \subset \mathbb{R}^{n}$ and $\Omega_{2} \subset \mathbb{R}^{m}$; this follows directly from the definition. Now considering the coderivative (2.5) for the multifunction $Q$ in (4.9) with $\operatorname{gph} Q=\Omega \times E$, we get the relationship

$$
z^{*} \in D^{*} Q(\bar{z},-b(\bar{z})-h(\bar{p}))\left(y^{*}\right) \Leftrightarrow z^{*} \in N(\bar{z} \mid \Omega) \&-y^{*} \in N(-b(\bar{z})-h(\bar{p}) \mid E) .
$$


This implies the equivalence between (7.2) and (7.6) for the multifunction $Q$ under consideration. According to the properties of $E$ and $\Omega$ assumed, one has

$$
\left\langle z_{1}-z_{2}, y_{1}-y_{2}\right\rangle=0 \text { for any } z_{1}, z_{2} \in \Omega \text { and } y_{1}, y_{2} \in E,
$$

i.e., the operator $Q$ in (4.9) is positive and negative monotone simultaneously. Therefore, we meet all the assumptions in Theorem 7.5 which ensures the sufficiency of condition (7.6) for the properties of the solution map being proved.

Now let us come back to the case of smooth generalized equations (5.1). We consider some additional assumptions when the sufficient condition (5.7) appears to be necessary and sufficient for the solution map (5.2) being locally single-valued and Lipschitz continuous.

Theorem 7.8. Let $f$ be strictly differentiable in $z$ at the point $(\bar{p}, \bar{z})$ satisfying the generalized equation (5.1). Assume that $Q$ is positive (negative) monotone and $\nabla_{z} f(\bar{p}, \bar{z})$ is positive (respectively, negative) semidefinite with respect to $\operatorname{Dom} Q$. Then:

(i) Condition (5.7) is necessary and sufficient for the solution map (5.2) being locally single-valued and Lipschitz continuous around $(\bar{p}, \bar{z})$ if one of the hypotheses $(\mathrm{H} 1)-(\mathrm{H} 3)$ in Theorem 5.8 is fulfilled.

(ii) For the separable form (6.12) of (5.1), the condition

$$
\left[0 \in(\nabla b(\bar{z}))^{*} y^{*}+D^{*} Q(\bar{z},-b(\bar{z})-h(\bar{p}))\left(y^{*}\right)\right] \Rightarrow y^{*}=0
$$

is necessary and sufficient for local single-valuedness and Lipschitz continuity of the solution map (7.1) around any point $(p, \bar{z})$ with $h(p)=h(\bar{p})$ if hypothesis $(\mathrm{H} 4)$ in Theorem 5.8 holds.

Proof. Note that condition (5.7) coincides with (7.5) and the solution map (7.1) is reduced to $(5.2)$ for the smooth separable generalized equations. Sufficiency parts in both assertions (i) and (ii) follow from Corollary 7.3. Now let us assume that the solution map (5.2) is locally single-valued and Lipschitz continuous around $(\bar{p}, \bar{z})$. Then it obviously will be pseudo-Lipschitzian around $(\bar{p}, \bar{z})$. According to assertion (i) in Theorem 5.8, condition (5.7) is fulfilled in the case under consideration when one of the hypotheses $(\mathrm{H} 1)-(\mathrm{H} 3)$ holds. This proves the necessity part in assertion (i) of Theorem 7.8 . The necessity part in assertion (ii) of this theorem is proved quite similarly by employing assertion (ii) in Theorem 5.8 .

Let us observe that, under the assumptions in Theorem 7.8, the solution map to the nonlinear generalized equation (5.1) is locally single-valued and Lipschitz continuous around $(\bar{p}, \bar{z})$ if and only if the solution map to the linearized equation (5.3) enjoys the same properties (cf. Corollary 5.9 for the pseudoLipschitzian property). One can easily formulate the corresponding analogues of Corollaries 5.11-5.16 providing necessary and sufficient conditions for the locally single-valuedness and Lipschitz continuity properties of the solution maps to some special generalized equations under additional monotonicity assumptions in Theorem 7.8.

Now we consider Robinson's strong reglarity property for smooth generalized equations and obtain its complete characterization using one of the results in Theorem 7.8. Let us take a point $(\bar{p}, \bar{z})$ satisfying $(5.1)$ and let us form the 
linearized operator $L(z)$ by formula (5.5). According to [29], the generalized equation

$$
0 \in f(\bar{p}, z)+Q(z)
$$

is said to be strongly regular at $\bar{z}$ if there exist neighborhoods $W$ of the origin in $\mathbb{R}^{m}$ and $V$ of $\bar{z}$ such that the restriction to $W$ of the operator $L^{-1} \cap V$ is a single-valued and Lipschitz continuous function. Here it is a characterization of the property introduced under monotonicity assumptions.

Corollary 7.9. Let $f$ be strictly differentiable in $z$ at $(\bar{p}, \bar{z})$. Assume that $Q$ is positive (negative) monotone and $\nabla_{z} f(\bar{p}, \bar{z})$ is positive (respectively, negative) semidefinite with respect to Dom $Q$. Then condition (5.7) is necessary and sufficient for the generalized equation (7.8) to be strongly regular at $\bar{z}$.

Proof. Let us consider the solution map (5.4) to the linearized generalized equation (5.3). Using the definitions, one can easily see that the strong regularity property of (7.8) at $\bar{z}$ is equivalent to the solution map $\Gamma$ in (5.4) being locally single-valued and Lipschitz continuous around $(0, \bar{z})$. It is obvious that the function

$$
\tilde{f}(y, z):=f(\bar{p}, \bar{z})+\nabla_{z} f(\bar{p}, \bar{z})(z-\bar{z})-y
$$

satisfies hypothesis $(\mathrm{H} 1)$ in Theorem 5.8 at $(0, \bar{z})$. Moreover, the matrix

$$
\nabla_{z} \tilde{f}(0, \bar{z})=\nabla_{z} f(\bar{p}, \bar{z})
$$

is either positive or negative semidefinite with respect to Dom $Q$ by virtue of our assumptions. Therefore, we can employ assertion (i) in Theorem 7.8 for the case of the generalized equation (5.3). According to this result, the solution map (5.4) to (5.3) is locally single-valued and Lipschitz continuous around $(0, \bar{z})$. This proves the strong regularity property of (7.8) at $\bar{z}$.

Remark 7.10. The strong regularity concept has been introduced and studied by Robinson [29] for the case of variational inequalities which corresponds to (7.8) with $Q(z)=\partial \delta(z, \Omega)$ for a convex set $\Omega$. This fundamental property of nonsingular solutions to variational inequalities appears to be equivalent to some other important properties (as Kojima's strong stability) and admits different characterizations; we refer to [16] for summarizing and recent developments. Various specifications and applications of strong regularity conditions in nonlinear programming and complementarity problems can be found in [7, $10,16,29,30]$ and elsewhere. It has been shown that such a strong regularity provides useful formulae for parametric sensitivity analysis ensuring, in particular, difference estimates between solutions to nonlinear problems and their linear approximations. The strong regularity assumption is basic in developing Newton-type numerical methods for solving variational inequalities and nonlinear complementarity problems; see [7, 16, 24] for references and more details. We hope that the results obtained in this paper will be useful to extend applications of strong regularity to more general settings.

Let us remind that perturbations problems in mathematical programming and complementarity as well as in general variational inequalities are imbedded in the framework of the generalized equations (5.1) with monotone multifunctions $Q$. Namely, such operators are maximal monotone being represented in the subdifferential form (4.25) with a convex function $\varphi$; see $\S 3$. Using this 
fact, one can formulate corollaries of all the results in this section for convex subdifferential mappings $Q(z)=\partial \varphi(z)$. These results are expressed in terms of the second order subdifferentials $\partial^{2,-} \varphi$ in (2.14) replacing the coderivative $D^{*} Q$ in the general statements above. Now we formulate, for instance, results in the case of smooth generalized equations with convex subdifferential mappings $Q$.

Corollary 7.11. Let $Q$ admit the subdifferential form (4.25) generated by a closed proper convex function $\varphi$ and let $f$ be strictly differentiable in $z$ at $(\bar{p}, \bar{z})$ where the gradient matrix $\nabla_{z} f(\bar{p}, \bar{z})$ is positive semidefinite with respect to Dom $Q$. Then condition (5.15) is sufficient for the solution map (5.2) being locally single-valued and Lipschitz continuous around $(\bar{p}, \bar{z})$ when $f$ is Lipschitzian in $p$ uniformly in $z$ around this point. Moreover, this condition is necessary and sufficient for the locally single-valuedness and Lipschitz continuity of $(5.2)$ around $(\bar{p}, \bar{z})$, when one of the hypotheses $(\mathrm{H} 1)-(\mathrm{H} 3)$ in Theorem 5.8 is fulfilled. In particular, condition (5.15) is always necessary and sufficient for the generalized equation (7.8) to be strongly regular at $\bar{z}$.

Proof. With the convex subdifferential mapping $Q(z)=\partial \varphi(z)$ in (5.1), we meet all the assumptions in Corollary 4.3, assertion (i) of Theorem 7.8, and Corollary 7.9 where condition (5.7) is reduced to (5.15) by virtue of (2.14).

Now let us return to Example 4.10 in $\S 4$ and let us use Corollary 7.11 for the linear generalized equation (4.1) equivalent to the perturbed variational inequality (4.28). In this way, one can conclude that the condition $a>0$ obtained is actually necessary and sufficient for the solution map to the variational inequality being single-valued and Lipschitz continuous around the origin.

Remark 7.12. If an extended-real-valued function $\varphi$ is concave, then the superdifferential mapping $Q(z)=\partial^{+} \varphi(z)=\partial \varphi(z)$ is negative monotone. In this case, we obtain an analogue of Corollary 7.11 where the matrix $\nabla_{z} f(\bar{p}, \bar{z})$ is negative semidefinite and (5.7) is replaced by the condition

$$
\left[0 \in\left(\nabla_{z} f(\bar{p}, \bar{z})\right)^{*} y^{*}+\partial^{2,+} \varphi(\bar{z},-f(\bar{p}, \bar{z}))\left(y^{*}\right)\right] \Rightarrow y^{*}=0
$$

in terms of the second order superdifferential in (2.15). One can easily formulate analogues of the other results in this section for the concave case under consideration.

If $\varphi: \mathbb{R}^{n} \times \mathbb{R}^{s} \rightarrow \overline{\mathbb{R}}$ is a closed proper saddle function (with $\varphi(z, w)$ convex in $z$ and concave in $w$ ), then its corresponding subdifferential [32, §35] forms a positive maximal monotone operator from $\mathbb{R}^{n} \times \mathbb{R}^{s}$ into itself. Therefore, this case also provides a special setting when one can employ general results in $\S 7$.

In conclusion we apply the results obtained in this section to state new conditions ensuring the existence of (single-valued) implicit functions with the local Lipschitzian property. Let us consider the equation

$$
f(p, z)=0 \text { with } f: \mathbb{R}^{k} \times \mathbb{R}^{n} \rightarrow \mathbb{R}^{n}
$$

and a point $(\bar{p}, \bar{z})$ satisfying (7.9). We say that (7.9) defines a locally singlevalued and Lipschitz continuous implicit function around $(\bar{p}, \bar{z})$ if there exist neighborhoods $U$ of $\bar{p}$ and $V$ of $\bar{z}$ as well as a Lipschitz continuous function $\tilde{z}: U \rightarrow V$ such that $\tilde{z}(p)$ is a unique solution to equation (7.9) for each $p \in U$ 
(so $\tilde{z}(\bar{p})=\bar{z})$. This means that the generalized equation (5.1) with $Q(z) \equiv$ 0 has the locally single-valued and Lipschitz continuous solution map around $(\bar{p}, \bar{z})$. Therefore, one can employ the results in this section for obtaining some generalizations of the classical implicit function theorem. Observe that the multifunction $Q(z) \equiv 0$ is positive and negative monotone simultaneously.

Corollary 7.13. Let $f$ be continuous in $(p, z)$ and locally Lipschitzian in $z$ uniformly in $p$ around the point $(\bar{p}, \bar{z})$ and let $f$ admit either a positive monotone or negative monotone strong approximation in $z$ at this point. Then equation (7.9) defines a locally single-valued and Lipschitz continuous implicit function if condition (6.30) is fulfilled. If, in particular, $f(\bar{p}, \cdot)$ is Lipschitzian around $\bar{z}$, then the sufficient condition (6.30) is equivalent to (6.31).

Proof. This follows from Theorem 7.1 when $Q(z) \equiv 0$; cf. Corollary 6.12.

Remark 7.14. According to (6.2) and Corollary 3.8(i), condition (6.30) is necessary and sufficient for any strong approximation $g$ of the function $f$ in $z$ at the point $(\bar{p}, \bar{z})$ to have the pseudo-Lipschitzian inverse $g^{-1}$ around $(g(\bar{z}), \bar{z})$. The monotonicity assumption in Corollary 7.13 ensures that $g^{-1}$ is actually single-valued around $g(\bar{z})$. Instead of the monotonicity, we can just assume that, in addition to (6.30), $f$ admits a strong approximation in $z$ at $(\bar{p}, \bar{z})$ with the (locally) single-valued inverse. Then Theorem 3.2 in Robinson [31] guarantees the existence of a locally single-valued and Lipschitz continuous implicit function (7.9) around $(\bar{p}, \bar{z})$. It happens, in particular, when $f(\bar{p}, \cdot)$ is Lipschitzian around $\bar{z}$ and (6.32) holds with $m=n$; see also Remark 6.13.

\section{ACKNOWLEDGMENTS}

The author gratefully acknowledges many fruitful discussions with Professor Terry Rockafellar on the material of this paper.

\section{REFERENCES}

1. J.-P. Aubin, Lipschitz behavior of solutions to convex minimization problems, Math. Oper. Res. 9 (1984), 87-111.

2. J.-P. Aubin and H. Frankowska, Set-valued analysis, Birkhäuser, Boston, MA, 1990.

3. J. M. Borwein and D. M. Zhuang, Verifiable necessary and sufficient conditions for regularity of set-valued and single-valued maps, J. Math. Anal. Appl. 134 (1988), 441-459.

4. F. H. Clarke, Optimization and nonsmooth analysis, Wiley, New York, 1983.

5. M. G. Crandall and P.-L. Lions, Viscosity solutions of Hamilton-Jacobi equations, Trans. Amer. Math. Soc. 277 (1983), 1-42.

6. A. L. Dontchev and W. W. Hager, Implicit functions, Lipschitz maps, and stability in optimization, Math. Oper. Res. (to appear).

7. M. S. Gowda and J.-S. Pang, Stability analysis of variational inequality and nonlinear complementarity problems, via the mixed linear complementarity problem and degree theory, Math. Oper. Res. (to appear).

8. P. T. Harker and J.-S. Pang, Finite-dimensional variational inequality and nonlinear comlementarity problems: a survey of theory, algorithms and applications, Math. Programming 48 (1990), 161-220.

9. A. D. Ioffe, Approximate subdifferentials and applications. I: The finite dimensional theory, Trans. Amer. Math. Soc. 281 (1984), 389-416.

10. A. J. King and R. T. Rockafellar, Sensitivity analysis for nonsmooth generalized equations, Math. Programming 55 (1992), 193-212. 
11. A. Kruger and B. Mordukhovich, Generalized normals and derivatives, and necessary conditions for extrema in nondifferentiable programming. I, Depon. VINITI No. 408-80 (1980, Russian).

12. __ Extrema! points and the Euler equation in nonsmooth optimization, Dokl. Akad. Nauk BSSR 24 (1980), 684-687. (Russian)

13. B. Kummer, An implicit function theorem for $C^{0,1}$-equations and parametric $C^{1,1}$. optimization, J. Math. Anal. Appl. 158 (1991), 35-46.

14. __ Lipschitzian inverse functions, directional derivatives, and applications in $C^{1,1}$ optimization, J. Optim. Theory Appl. 70 (1991), 561-582.

15. J. Kyparisis, Parametric variational inequalities with multivalued solution sets, Math. Oper. Res. 17 (1992), 341-364.

16. J. Liu, Nonsingular solutions of finite-dimensional variational inequalities: theory and methods, preprint, 1992.

17. B. Mordukhovich, Maximum principle in the problem of time-optimal control with nonsmooth constraints, J. Appl. Math. Mech. 40 (1976), 960-969.

18. __ Metric approximations and necessary optimality conditions for general classes of nonsmooth extremal problems, Soviet Math. Dokl. 22 (1980), 526-530.

19. __ Approximation methods in problems of optimization and control, Nauka, Moscow, 1988. (Russian; English transl. to appear in Wiley)

20. Sensitivity analysis in nonsmooth optimization, Theoretical Aspects of Industrial Design (D. A. Field and V. Komkov, eds.), SIAM Proc. Appl. Math., vol. 58, 1992, pp. 32-46.

21. _ On variational analysis of differential inclusions, Optimization and Nonlinear Analysis (A. Ioffe, L. Marcus, and S. Reich, eds.), Pitman Res. Notes Math. Ser., 224, Pitman, 1992, pp. 199-213.

22. __ Complete characterization of openness, metric regularity, and Lipschitzian properties of multifunctions, Trans. Amer. Math. Soc. 340 (1993), 1-36.

23. _ Lipschitzian stability of constraint systems and generalized equations, Nonlinear Anal. 22 (1994).

24. J.-S. Pang, A degree-theoretic approach to parametric nonsmooth equations with multivalued perturbed solutions sets, Math. Programming 62 (1993), 359-383.

25. J.-P. Penot, Metric regularity, openness and Lipschitzian behavior of multifunctions, Nonlinear Anal. Theory Methods Appl. 13 (1989), 629-643.

26. Y. Qiu and T. L. Magnati, Sensitivity analysis for variational inequalities, Math. Oper. Res. 17 (1992), 61-76.

27. S. M. Robinson, Stability theory for systems of inequalities. II: Differentiable nonlinear systems, SIAM J. Numer. Anal. 13 (1976), 497-513.

28. _ Generalized equations and their solutions, part I: basic theory, Math. Programming Stud. 10 (1979), 128-141.

29. __ Strongly regular generalized equations, Math. Oper. Res. 5 (1980), 43-62.

30. _ Generalized equations, Math. programming: The State of Art (A. Bachem, M. Grötschel, and B. Korte, eds.), Springer-Verlag, Berlin and New York, 1982, pp. 346-367.

31. An implicit-function theorem for a class of nonsmooth functions, Math. Oper. Res. 16 (1991), 292-309.

32. R. T. Rockafellar, Convex analysis, Princeton Univ. Press, Princeton, NJ, 1970.

33. The theory of subgradients and its applications to optimization: convex and nonconvex functions, Heldermann, 1981.

34. Lipschitzian properties of multifunctions, Nonlinear Anal. Theory Methods Appl. 9 (1985), 867-885.

35. Maximal monotone relations and the second derivatives of nonsmooth functions, Ann. Inst. H. Poincaré. Anal. Non Linéaire 2 (1985), 167-184.

36. R.T. Rockafellar and R. J.-B. Wets, Variational analysis, Springer-Verlag (to appear). 
37. A. Shapiro, Sensitivity analysis of parametric programs via generalized equations, preprint, 1992.

38. L. Thibault, Subdifferentials of compactly Lipschitzian functions, Ann. Math. Pura Appl. (4) 125 (1980), 157-192.

Department of Mathematics, Wayne State University, Detroit, Michigan 48202-9861

E-mail address: boris@math . wayne.edu 